\title{
Oxidized Alginate Dopamine Conjugate: In Vitro Characterization for Nose-to-Brain Delivery Application
}

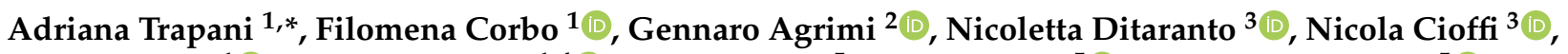

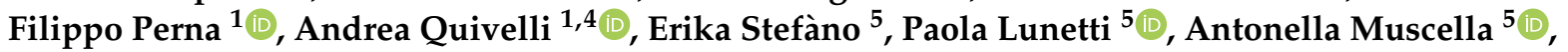

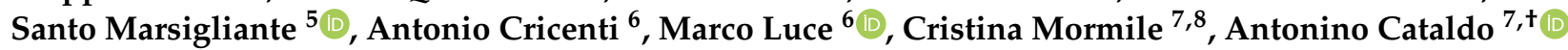 \\ and Stefano Bellucci ${ }^{7, *}$ (])
}

check for updates

Citation: Trapani, A.; Corbo, F.; Agrimi, G.; Ditaranto, N.; Cioffi, N.; Perna, F.; Quivelli, A.; Stefàno, E.;

Lunetti, P.; Muscella, A.; et al.

Oxidized Alginate Dopamine

Conjugate: In Vitro Characterization for Nose-to-Brain Delivery

Application. Materials 2021, 14, 3495.

https://doi.org/10.3390/ma14133495

Academic Editor: Dokyoung Kim

Received: 20 May 2021

Accepted: 18 June 2021

Published: 23 June 2021

Publisher's Note: MDPI stays neutral with regard to jurisdictional claims in published maps and institutional affiliations.

Copyright: (c) 2021 by the authors. Licensee MDPI, Basel, Switzerland. This article is an open access article distributed under the terms and conditions of the Creative Commons Attribution (CC BY) license (https:/ / creativecommons.org/licenses/by/ $4.0 /)$.
1 Department of Pharmacy-Drug Sciences, University of Bari “Aldo Moro", I-70125 Bari, Italy; filomena.corbo@uniba.it (F.C.); filippo.perna@uniba.it (F.P.); andrea.quivelli@uniba.it (A.Q.)

2 Department of Biosciences, Biotechnologies and Biopharmaceutics, University of Bari "Aldo Moro", I-70125 Bari, Italy; gennaro.agrimi@uniba.it

3 Dipartimento di Chimica and CSGI-Bari Unit, Università degli Studi di Bari Aldo Moro, I-70125 Bari, Italy; nicoletta.ditaranto@uniba.it (N.D.); nicola.cioffi@uniba.it (N.C.)

4 Consorzio C.I.N.M.P.I.S., Via E. Orabona 4, I-70125 Bari, Italy

5 Dipartimento Scienze e Tecnologie Biologiche e Ambientali, University of Salento, I-73100 Lecce, Italy; erika.stefano@unisalento.it (E.S.); paola.lunetti@unisalento.it (P.L.); antonella.muscella@unisalento.it (A.M.); santo.marsigliante@unisalento.it (S.M.)

6 ISM-CNR, Via del Fosso del Cavaliere 100, I-00133 Rome, Italy; antonio.cricenti@artov.ism.cnr.it (A.C.); marco.luce@artov.ism.cnr.it (M.L.)

7 Istituto Nazionale di Fisica Nucleare-Laboratori Nazionali di Frascati, Via Enrico Fermi 54, Frascati, I-00044 Rome, Italy; c.mormile@gmail.com (C.M.); antonino.cataldo@lnf.infn.it (A.C.)

8 Department of Chemical Science and Technologies, University of Rome Tor Vergata, Via della Ricerca Scientifica 1, I-00133 Rome, Italy

* Correspondence: adriana.trapani@uniba.it (A.T.); stefano.bellucci@lnf.infn.it (S.B.)

+ Present address: ENEA Casaccia, Laboratorio Tecnologie per la Dinamica delle Strutture e la Prevenzione del Rischio Sismico e Idrogeologico (DISPREV), 00133 Rome, Italy.

Abstract: Background: The blood-brain barrier (BBB) bypass of dopamine (DA) is still a challenge for supplying it to the neurons of Substantia Nigra mainly affected by Parkinson disease. DA prodrugs have been studied to cross the BBB, overcoming the limitations of DA hydrophilicity. Therefore, the aim of this work is the synthesis and preliminary characterization of an oxidized alginatedopamine (AlgOX-DA) conjugate conceived for DA nose-to-brain delivery. Methods: A Schiff base was designed to connect oxidized polymeric backbone to DA and both AlgOX and AlgOX-DA were characterized in terms of Raman, XPS, FT-IR, and ${ }^{1} \mathrm{H}$ - NMR spectroscopies, as well as in vitro mucoadhesive and release tests. Results: Data demonstrated that AlgOX-DA was the most mucoadhesive material among the tested ones and it released the neurotransmitter in simulated nasal fluid and in low amounts in phosphate buffer saline. Results also demonstrated the capability of scanning near-field optical microscopy to study the structural and fluorescence properties of AlgOX, fluorescently labeled with fluorescein isothiocyanate microstructures. Interestingly, in SH-SY5Y neuroblastoma cell line up to $100 \mu \mathrm{g} / \mathrm{mL}$, no toxic effect was derived from AlgOX and AlgOX-DA in $24 \mathrm{~h}$. Conclusions: Overall, the in vitro performances of AlgOX and AlgOX-DA conjugates seem to encourage further ex vivo and in vivo studies in view of nose-to-brain administration.

Keywords: dopamine; oxidized alginate; mucoadhesion; microscopy; cell viability

\section{Introduction}

Parkinson's disease (PD), the second most common neurological disorder (ND) after Alzheimer disease, is mainly characterized by the progressive loss of dopaminergic neurons in the Substantia Nigra, with a consequent dysregulation of motor activity [1]. From a clinical point of view, the death of dopaminergic neurons brings about classical PD symptoms, 
including bradykinesia, muscular rigidity, and tremors. On the other hand, the histopathological hallmark of the disease is the presence of Lewy bodies, i.e., abnormal aggregates of proteins of which $\alpha$-synuclein is the main component [2].

One of the main problems to face in PD treatment, as well as in other NDs, is the transport of therapeutic agents across the blood-brain barrier (BBB), which, even though it is permeable, still limits the entrance of molecules into brain, which not only may cause neuronal damage, but also may have therapeutic usefulness [3]. Therefore, a very critical issue to treat NDs is to bypass the BBB.

In recent years, several research groups have highlighted that administration via the intranasal route of neurotherapeutics constitutes an advantageous approach to bypass the $\mathrm{BBB}$, thus enabling direct access to the brain by olfactory and trigeminal neural connections between the nose and brain [4-7]. The most relevant advantage of using nose-to-brain drug administration is the minimally invasive feature of this pathway, leading to a high patient compliance; hence, the design of nasal formulations is crucial in order to address the active principles to the site of action [7]. For instance, the anatomic features of the olfactory cleft at the roof of the nasal cavity require that excipients with good mucoadhesion properties be good candidates to prolong the residence time at the mucosa so that drug bioavailability can be increased [7]. Among mucoadhesive polymers for nasal delivery, chitosan, carbopol, carboxymethylcellulose, polyacrylic acid, and others have been extensively described. In particular, semisolid formulations, such as hydrogels, have demonstrated their suitability in intranasal administration. Due to their molecular structure, hydrogels exhibit distinct advantages, being biocompatible, having low toxicity, and hydrophilic delivery systems. Moreover, their drug release kinetics can be tuned by controlling the crosslinking degree; some hydrogels can possess interesting features, including P-glycoprotein efflux pump (P-gp) inhibition and further biomedical applications [8-10]. In addition to hydrogels, nanoparticulate systems, such as polymeric nanoparticles, polymeric micelles and lipidbased formulations, have also been used in nose-to-brain delivery [7,11-13].

Currently, the main objective of PD therapy is to compensate for the loss of dopaminergic neurons and to restore suitable amounts of the neurotransmitter, dopamine (DA), which cannot cross the BBB. This approach is denoted as a "dopamine replacement strategy" and has been evaluated by loading different nanocarriers with both the free neurotransmitter (or its precursor L-dopa) and dopaminergic drugs [14-18]. A potential drawback of this strategy is the premature leakage of the therapeutic cargo from the nanocarrier, which can be minimized by linking the therapeutic agent to a polymeric backbone using a cleavable chemical bond. The resulting macromolecular conjugates possess several advantages, including a controlled release kinetic, prolonged circulation, and improved mucoadhesive properties [19-21]. In addition, they may provide free neurotransmitter- or dopaminergic drug-loaded nanoparticulate systems that are potentially able to cross the $\mathrm{BBB}$ via endocytosis $[22,23]$.

The aim of the present work was to synthesize and characterize, from a physicochemical point of view, a novel macromolecular conjugate of DA, i.e., oxidized alginate (AlgOX) imine conjugate (AlgOX-DA), for potential intranasal DA delivery. Alginates (Alg), a class of natural-origin polysaccharides, are anionic linear block copolymers comprised of 1,4linked -D-mannuronic acid (M) and -L-guluronic acid (G) (Figure 1), from which physical hydrogels are formed using solutions containing $\mathrm{Ca}^{2+}$ and $\mathrm{Ba}^{2+}$ divalent cations [24]. However, Alg-derived hydrogels are limited by their slow degradability in vivo, and oxidation of Alg leads to an increased biodegradability [24]. Moreover, a further advantage of AlgOX compared to Alg is that the former possesses additional organic functions that can be exploited in the conjugation process. For instance, in the oxidation process, two aldehyde groups are created, which can be useful in the conjugation reaction with a primary amino group containing substances [25]. Therefore, most interest has been focused on AlgOX for several biomedical applications. In this work, in addition to the synthesis of AlgOX-DA imine conjugate and its spectroscopic characterization, we describe the evaluation of its 
mucoadhesive properties, DA release kinetic in simulated nasal fluid and in phosphate buffer saline, as well as cytotoxicity by the human neuroblastoma SH-SY5Y cells line.

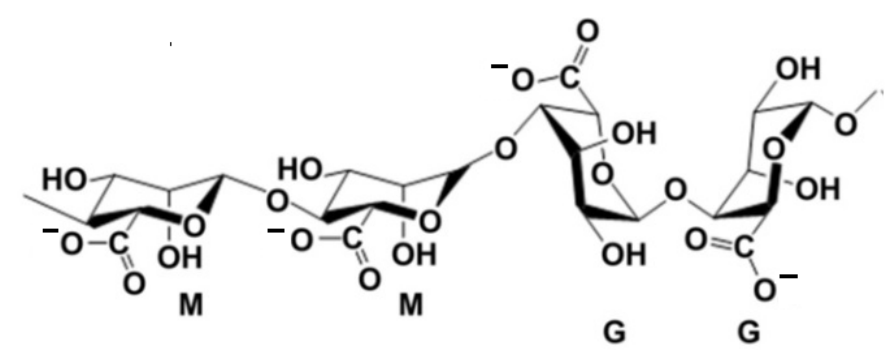

Figure 1. Chemical structure of alginate.

\section{Materials and Methods}

Sodium alginate $(\mathrm{NaAlg}, \mathrm{M} / \mathrm{G}=1.49$; Molecular weight $=396 \mathrm{kDa})$, sodium periodate $\left(\mathrm{NaIO}_{4}\right)$, dopamine hydrochloride (DA), polyethylene glycol (Molecular weight $\left.=200 \mathrm{~g} / \mathrm{mol}\right)$, triethylamine (TEA), porcine stomach mucin (type II, bound sialic acid $\sim 1 \%$ ), and fluorescein 5(6)-isothiocyanate (FITC) were provided from Sigma-Aldrich (Milan, Italy). Hydroxyethyl cellulose (HEC, Natrosol 250) was purchased from Aakon Polichimica (Milan, Italy). According to the manufacturer instructions, a solution of HEC at a concentration of $2 \%$ in water provided a viscosity value equal to $5500 \mathrm{mPa} \cdot \mathrm{s}$. Dialysis tubes with a MWCO $3500 \mathrm{Da}$ and 1200-14,000 Da were purchased from Spectra Labs (Rome, Italy). Throughout this work, double distilled water was used. All other chemicals used were of reagent grade.

\subsection{Synthesis of Oxidized Alginate and Oxidized Alginate-DA}

A total of $500 \mathrm{mg}$ of NaAlg was dissolved in $5 \mathrm{~mL}$ of ethanol and then $530 \mathrm{mg}$ of $\mathrm{NaIO}_{4}$, was added and dissolved in $5 \mathrm{~mL}$ of distilled water under stirring at r.t. and under dark conditions for $24 \mathrm{~h}$. To quench the reaction, a few drops of polyethylene glycol were added, leaving the solution under stirring for a further $30 \mathrm{~min}$.

The resulting product was purified by solubilization/precipitation according to the following procedure. Distilled water $(5 \mathrm{~mL})$ was added to the reaction mixture in order to solubilize the $\mathrm{AlgOX}$, then $\mathrm{NaCl}(0.20 \mathrm{~g})$ dissolved in ethanol $(5 \mathrm{~mL})$ was added to precipitate the NaAlg which was collected by filtration This solubilization/precipitation procedure was repeated two more times; the first by dissolving the filtrate AlgOX in distilled water $(5 \mathrm{~mL})$ and then adding $\mathrm{NaCl}(0.06 \mathrm{~g})$ dissolved in ethanol $(5 \mathrm{~mL})$ to precipitate the AlgOX. Finally, dissolution of the filtrate in distilled water $(5 \mathrm{~mL})$ and addition of $\mathrm{NaCl}(0.03 \mathrm{~g})$ and acetone $(10 \mathrm{~mL})$ occurred to precipitate AlgOX. Ethanol $(5 \mathrm{~mL})$ was poured to wash the collected final precipitate under stirring for $15 \mathrm{~min}$ and then the precipitate was dried at $45^{\circ} \mathrm{C}$ under reduced pressure. A constant weight was obtained [26].

$$
\text { FT-IR (KBr) } \vee\left(\mathrm{cm}^{-1}\right): 3435(\mathrm{OH}), 2915(\mathrm{HC}=\mathrm{O}), 1622(\mathrm{C}=\mathrm{O}) 1388(\mathrm{OH}) \mathrm{cm}^{-1} .
$$

The AlgOX-DA imine conjugate was prepared according to the synthetic procedure outlined in Scheme 1, following Gao et al.'s methodology [27]. A solution of AlgOX (25 mg) in distilled water $(3 \mathrm{~mL}, \mathrm{pH} 4)$ was poured into a solution of DA $(68 \mathrm{mg})$ and TEA $(55 \mu \mathrm{L})$ in distilled water $(1 \mathrm{~mL})$, in a light protected vessel under a nitrogen atmosphere. Then, the mixture underwent magnetic stirring in the dark for $6 \mathrm{~h}$. The obtained dispersion was dialyzed in dialysis tubes (MWCO $=12,000-14,000 \mathrm{Da}$ ) and lyophilized for $96 \mathrm{~h}$ (Lio5Pascal, Italy) to obtain a brown powder as product in a yield of $62.8 \%$.

AlgOX-DA: FT-IR (KBr) $v\left(\mathrm{~cm}^{-1}\right)$ : $3439(\mathrm{OH}), 2917(\mathrm{HC}=\mathrm{O}), 2073$ (aliphatic $\left.\mathrm{CH}\right), 1636(\mathrm{C}=\mathrm{N}) \mathrm{cm}^{-1}$. 


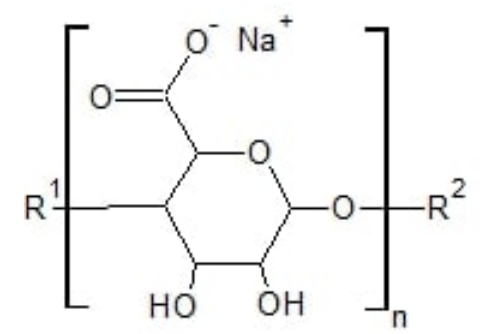

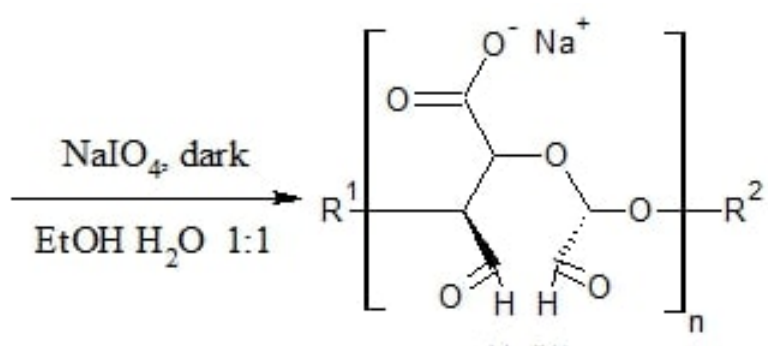

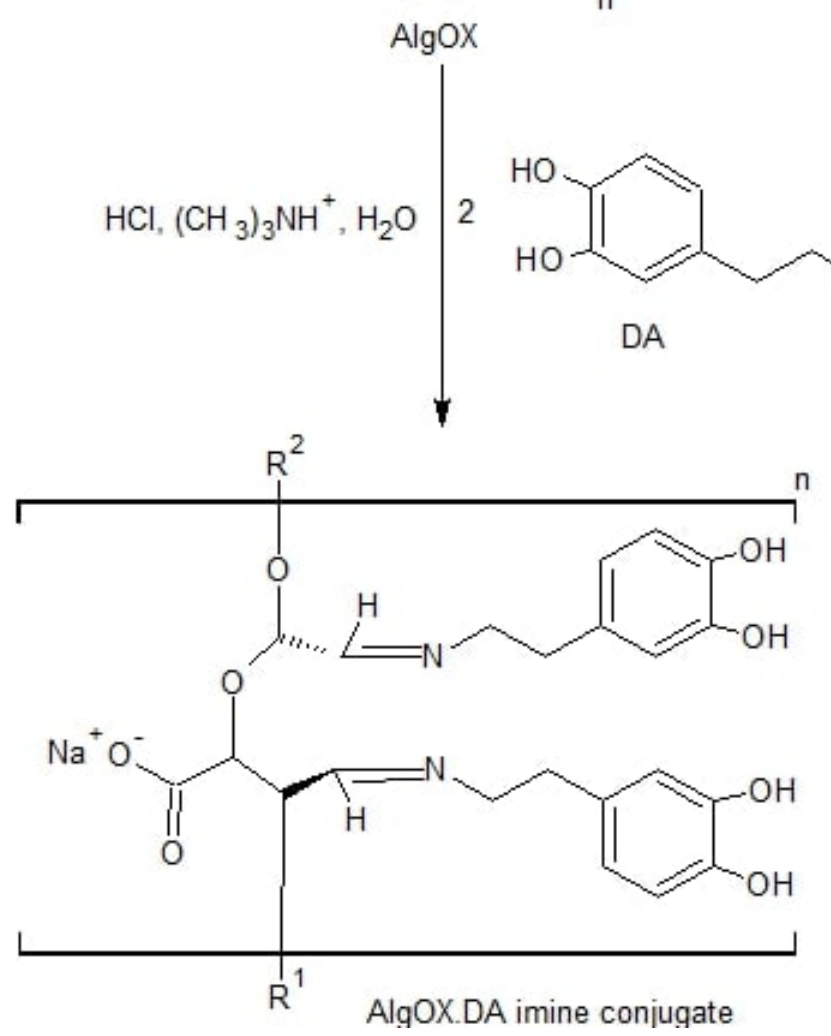

Scheme 1. The synthetic pathway followed to prepare AlgOX-DA imine conjugate.

\subsection{Quantitative Analysis of $D A$}

The quantification of DA was performed using HPLC, as previously reported [28]. Precisely, the mobile phase was a mixture of $0.02 \mathrm{M}$ potassium phosphate buffer, $\mathrm{pH} 2.8$ : $\mathrm{CH}_{3} \mathrm{OH} 70: 30(\mathrm{v} / \mathrm{v})$, and the isocratic elution of the column occurred at a flow rate of $0.7 \mathrm{~mL} / \mathrm{min}$. In the HPLC chromatograms, the retention times of DA and AlgOX-DA were equal to 5.5 and $4.9 \mathrm{~min}$, respectively.

\subsection{Spectroscopic Characterization}

\subsubsection{UV-Vis}

For pure DA, AlgOX and AlgOX-DA, UV-vis spectra were acquired with a PerkinElmer Lambda Bio 20 spectrophotometer at a wavelength range of 230-340 nm. Prior to acquiring the spectra, pure DA aqueous solution was prepared at $0.05 \mathrm{mg} / \mathrm{mL}$ at $25^{\circ} \mathrm{C}$, and $\mathrm{AlgOX}$ and AlgOX-DA were dissolved, obtaining the final concentrations of $1 \mathrm{mg} / \mathrm{mL}$ and $0.25 \mathrm{mg} / \mathrm{mL}$, respectively. A slight sonication was required for complete dissolution for AlgOX-DA.

\subsubsection{FT-IR Spectroscopy}

FT-IR spectra were obtained with KBr discs using a Perkin Elmer 1600 FT-IR spectrometer (Perkin Elmer, Milan Italy). The analysis was carried out at room temperature (r.t.) in the range of $4000-400 \mathrm{~cm}^{-1}$ at a resolution of $1 \mathrm{~cm}^{-1}$ for the following samples: pure DA, AlgOX, and AlgOX-DA. 


\subsubsection{Raman Spectroscopy}

To record the Raman spectra an InVia microscope (Renishaw, Wotton-under-Edge, UK) was employed with a laser source set at a wavelength of $532 \mathrm{~nm}$ in the presence of a $100 \times$ objective. The specimens were placed onto a microscope slide. The range examined was $200-4200 \mathrm{~cm}^{-1}$ using a 1800 (L)/mm grating.

\subsubsection{Scanning Electron Microscopy (SEM) and X-ray Energy Dispersive (EDX) Analysis}

SEM and EDX analyses were carried out using a VegalI microscope (Tescan, Czech Republic), with a Quantax elemental detector (Bruker, Billerica, MA, USA). The specimens were placed onto a microscope stub and a sputter coater (Cressington, UK) was used to cover specimens with a thin gold layer. Then, the specimens were introduced into the SEM chamber and analyses were performed using a high voltage of $20 \mathrm{kV}$.

\subsubsection{X-ray Photoelectron Spectroscopy (XPS) Analysis}

To determine XPS spectra, a Versa Probe II scanning XPS microprobe spectrometer (Physical Electronics GmbH, Feldkirchen near Munich, Germany), equipped with a monochromatized $\mathrm{Al} \mathrm{K \alpha}$ source $(X$-ray spot $=200 \mu \mathrm{m})$, with a working power of $50.3 \mathrm{~W}$, was used. In order to acquire wide scans and detailed spectra, the fixed analyzer transmission (FAT) mode, with a pass energy of $117.40 \mathrm{eV}$ and $29.35 \mathrm{eV}$, respectively, was employed. Charge compensation (1.1 V $20.0 \mu \mathrm{A})$ was obtained using an electron gun. Data processing was performed using MultiPak software v. 9.9.0.8 (Physical Electronics GmbH, Feldkirchen near Munich, Germany).

\subsection{NMR Spectroscopy}

A Bruker $500-\mathrm{Hz}$ spectrometer allowed the recording of ${ }^{1} \mathrm{H}-\mathrm{NMR}$ spectra and parts per million $(\delta)$ was used to report chemical shifts. Spectra were processed using Mnova (Mestrelab Research).

To allow the determination of AlgOX-DA composition via ${ }^{1} \mathrm{H}$ NMR spectrometry, dissolution in $\mathrm{D}_{2} \mathrm{O}$ was performed for this sample at a concentration of $6 \mathrm{mg} / \mathrm{mL}$ and heated at $40{ }^{\circ} \mathrm{C}$ for few minutes before the analysis.

\subsection{Differential Scanning Calorimetry (DSC)}

Prior to perform thermal analyses, melting point determination for AlgOX and AlgOXDA occurred via the use of the capillary method (Stuart Scientific SMP3 electrothermal apparatus, Bibby Scientific, Milan, Italy). DSC thermograms were obtained for DA, AlgOX, and AlgOX-DA working with a Mettler Toledo DSC 822e STARe 202 System equipped with DSC MettlerSTARe Software (version II, Mettler-Toledo, Milan, Italy). About $5 \mathrm{mg}$ of each sample was placed in an aluminum pan and hermetically sealed. The scanning rate was set to $5{ }^{\circ} \mathrm{C} / \mathrm{min}$ under an indium atmosphere $(99.9 \%)$ and adopted for DSC calibration following the procedure of MettlerSTARe Software. Each experiment was carried out in triplicate.

\subsection{Determination of Substitution Degree (DS) of AlgOX-DA}

For substitution degree (DS) determination, the AlgOX-DA conjugate was subjected to acid hydrolysis. Briefly, $2 \mathrm{mg}$ of AlgOX-DA conjugate were weighed and dissolved in $\mathrm{HCl} 1 \mathrm{~N}$ (pH 1) under stirring and light protection at r.t. for $3 \mathrm{~h}$. Afterwards, the yellow liquid was collected, which was then centrifuged $(16,000 \times g$, for $45 \mathrm{~min}$, Eppendorf 5415D, Germany) prior to performing HPLC analyses (Section 2.2) of the obtained supernatant. DS was calculated as mg DA/mg AlgOX-DA imine conjugate.

\subsection{Mucoadhesion Tests}

The evaluation of the mucoadhesive properties of the AlgOX and AlgOX-DA conjugate in simulated nasal fluid (SNF) was based on an in vitro method which used turbidimetric 
measurements, as described previously [21,28]. SNF composition included $\mathrm{CaCl}_{2} \cdot 2 \mathrm{H}_{2} \mathrm{O}$ (0.32 mg/mL), $\mathrm{KCl}(1.29 \mathrm{mg} / \mathrm{mL})$, and $\mathrm{NaCl}(7.45 \mathrm{mg} / \mathrm{mL})$ at $\mathrm{pH} 6.0$ [29].

Freshly prepared mucin dispersions in SNF $(0.5 \mathrm{mg} / \mathrm{mL})$ were incubated at $37^{\circ} \mathrm{C}$ under stirring $(150 \times g)$ for $24 \mathrm{~h}$ before starting the experiments. Separately, all samples were dispersed in SNF, providing a final concentration of $0.04 \%(w / v)$. To $6 \mathrm{~mL}$ of mucin dispersion in SNF, $6 \mathrm{~mL}$ of sample dispersion was added and the turbidity of the corresponding mixtures, incubated at $37^{\circ} \mathrm{C}$ and under stirring $(150 \times g)$, was determined at a wavelength of $650 \mathrm{~nm}$ using a Perkin-Elmer Lambda Bio 20 spectrophotometer at different time points (i.e., $0,2,5,7$ and $24 \mathrm{~h})$. HEC $(0.4 \mathrm{mg} / \mathrm{mL}$ in SNF) was employed as positive control. All experiments were carried out in triplicate.

\subsection{In Vitro Release Studies}

A total of 10-12 mg of AlgOX-DA (corresponding to $0.3-0.5 \mathrm{mg}$ of DA) were collected in a dialysis bag (MWCO $3500 \mathrm{Da}$ ) containing $1.5 \mathrm{~mL}$ of double distilled water and then the dialysis bag was dispersed in $20 \mathrm{~mL}$ of SNF (or PBS) at $37 \pm 0.1^{\circ} \mathrm{C}$ in an agitated $(40 \times \mathrm{g} / \mathrm{min})$ water bath (Julabo, Milan, Italy). At scheduled time-points, $0.8 \mathrm{~mL}$ was withdrawn from the receiving medium and $0.8 \mathrm{~mL}$ of fresh medium was used to maintain sink conditions. Each sample underwent centrifugation $(16,000 \times g, 45 \mathrm{~min}$, Eppendorf 5415D, Germany), and the concentrations of DA were quantified in the resulting supernatant by HPLC, as reported in Section 2.2. For the cumulative release calculation, an approach reported in the literature was followed [30]. In details, the calculation of the accumulated release was based on the following equation:

$$
\mathrm{Q}_{\mathrm{n}}=\mathrm{C}_{\mathrm{n}} \times \mathrm{V}_{0}+\left(\mathrm{C}_{1}+\mathrm{C}_{2}+\mathrm{C}_{3}+\ldots . \mathrm{C}_{\mathrm{n}-1}\right) \times \mathrm{V}
$$

$\mathrm{Q}_{\mathrm{n}}$ is the cumulative release of the $n$-th sampling point, $\mathrm{C}$ represents the solution concentration in the SNF (or PBS), $\mathrm{C}_{\mathrm{n}}$ is the concentration in the SNF (or PBS) of the $n$-th sampling point, $\mathrm{V}_{0}$ is the total volume of release medium, and $\mathrm{V}$ is the volume of each sample withdrawn.

All release experiments were carried out in triplicate.

\subsection{Preparation of Fluorescent AlgOX}

Following a previous protocol [31], after dissolving $30 \mathrm{mg}$ of AlgOX in $3 \mathrm{~mL}$ of double distilled water, the $\mathrm{pH}$ value was adjusted to 5 by dropping in $\mathrm{HCl} 0.01 \mathrm{~N}$. Then, $1 \mathrm{~mL}$ of an ethanolic solution of FITC $(5 \mathrm{mg} / \mathrm{mL})$ was added and stirring was applied for $24 \mathrm{~h}$ under dark conditions at r.t. The mixture was then dialyzed in water using dialysis tubing (SpectraPore ${ }^{\circledR}$ Dialysis MWCO 12,000-14,000 Da) for 3 days and then freeze dried for $72 \mathrm{~h}$ (Lio Pascal 5P, Italy).

To determine the labeling efficiency of the resulting FITC-AlgOX conjugate, the fluorescence intensity of FITC-AlgOX dissolved in $0.1 \mathrm{M}$ phosphate buffer, $\mathrm{pH}$ 8.0, was determined at a concentration of $2 \mu \mathrm{g} / \mathrm{mL}$. The labeling efficiency (\%) was the percent weight of FITC to weight of the FITC-polymer. The fluorometer was calibrated with standard solutions of 1 to $140 \mathrm{ng} / \mathrm{mL}$ of FITC in phosphate buffer, $\mathrm{pH}$ 8.0, arising from dilution in such a buffer of a stock solution of $100 \mu \mathrm{g} / \mathrm{mL}$ FITC in methanol (excitation and emission wavelengths of 488 and $525 \mathrm{~nm}$, respectively; slits, $2.5 \mathrm{~cm}$ ).

\subsection{Photon Correlation Spectroscopy and SNOM Visualization}

Particle size and polydispersity index for AlgOX and AlgOX-DA were determined $\backslash$ using a Zetasizer Nano ZS (ZEN 3600, Malvern, UK) apparatus using the photon correlation spectroscopy (PCS) mode. Particle size and PDI were measured at concentrations of $1 \mathrm{mg} / \mathrm{mL}$ and $0.25 \mathrm{mg} / \mathrm{mL}$ for aqueous AlgOX and AlgOX-DA, respectively, introducing $1 \mathrm{~mL}$ of each sample into the cuvette.

For SNOM visualization, the apparatus setup employed consisted of a hybrid inverted/SNOM, a home-built SNOM connected to a conventional inverted optical microscope [32]. The instrument allowed the collection of topographic images simultaneously 
with optical data; both transmittance and fluorescence were recorded and the fluorophore signal had a typical absorbing peak above $400 \mathrm{~nm}$ and a wide-band emission above $500 \mathrm{~nm}$. The $405 \mathrm{~nm}$ laser line was coupled in the Al-covered silica optical fiber and the fluorescence and transmitted light were collected by the $20 \times$ lenses of an Olympus microscope through a high-pass filter. The fluorescence signal was collected above $500 \mathrm{~nm}$. This system worked with the inverted microscope to determine the features of the solutions of FITC-AlgOX ( $1 \mathrm{mg} / \mathrm{mL}$ and $0.01 \mathrm{mg} / \mathrm{mL}$ in water), to perform a far-field characterization and to pin-point the location where the high resolution SNOM measurements should be carried out. For analysis, FITC-AlgOX samples were deposited onto a glass substrate by means of a micropipette, dried, and examined using shear-force and fluorescence SNOM nanospectroscopy. Data were collected from several sample areas using different sets of experiments, and then analyzed using Gwyddion software.

\subsection{Cell Culture}

SH-SY5Y cell line was grown in DMEM medium (Sigma, St. Louis, MO, USA) supplemented with $10 \%$ heat-inactivated fetal bovine serum (FBS), glutamine $(2 \mathrm{mM})$, penicillin $(100 \mathrm{U} / \mathrm{mL})$ and streptomycin $(100 \mathrm{mg} / \mathrm{mL})$ in a humidified incubator with $5 \% \mathrm{CO}_{2}$ in air at $37^{\circ} \mathrm{C}$.

\subsection{Cytotoxicity Assays}

Cell proliferation of SH-SY5Y inhibition was investigated according to sulforhodamine B (SRB) assay. Briefly, cells at $70-80 \%$ confluency were treated with trypsin $(0.25 \%$ trypsin with $1 \mathrm{mM}$ EDTA), washed, and resuspended in growth medium; $100 \mu \mathrm{L}$ of cell suspension $\left(10^{5}\right.$ cells $\left./ \mathrm{mL}\right)$ was added to each well of a 96-well plate. After overnight incubation, cells were treated with different concentrations of AlgOX (1-400 $\mu \mathrm{g} / \mathrm{mL})$, AlgOX-DA, and DA for $24 \mathrm{~h}$. After treatment, cells were fixed in $10 \%$ trichloroacetic-acid (TCA) and stained with $0.4 \%(w / v)$ SRB dissolved in $1 \%$ acetic acid. SRB was removed and the plates were washed four times with $1 \%$ acetic acid to remove unbound dye before air-drying. Bound SRB was dissolved in $200 \mu \mathrm{L}$ of $10 \mathrm{mM}$ unbuffered Tris-based solution and plates were left on a plate shaker for at least $10 \mathrm{~min}$. Absorbance was measured in a 96-well plate reader at $492 \mathrm{~nm}$.

The absorbance ratio of treated to untreated cells allowed the calculation of the percentage of cell survival. The data presented are means \pm standard deviation (S.D.) from eight replicates of five independent experiments. Viable cells were also counted by the trypan blue exclusion assay and light microscopy.

\subsection{Statistics}

For release studies and mucoadhesion tests, statistical analyses were carried out using Prism Version 4, GraphPad Software Inc. (San Diego, CA, USA). Data were expressed as either mean $\pm \mathrm{SD}$. Multiple comparisons were based on one-way analysis of variance (ANOVA) with the either Bonferroni's or Tukey's post hoc test, and differences were considered significant when $p<0.05$.

For biological studies, experimental points represented means \pm SD of three to five independent experiments, performed in eight replicates, undergoing statistical evaluation using the ANOVA test. Where indicated, post hoc tests (Bonferroni/Dunn) were also performed.

\section{Results}

\subsection{Synthesis of AlgOX and AlgOX-DA and Raman Spectroscopy Identification}

The synthesis of AlgOX was carried out by oxidizing NaAlg with $\mathrm{NaIO}_{4}$, and oxidation occurred over $24 \mathrm{~h}$ on the $-\mathrm{OH}$ groups at $\mathrm{C} 2$ and $\mathrm{C} 3$ of the of the $\mathrm{M}$ and $\mathrm{G}$ residues with main oxidation of the $G$ residues [33]. Being a photosensitive reaction, the procedure was performed in a container with darkened walls [26], where, through the use of a 1:1 mixture (water:ethanol, $v / v$ ), the final yield achieved was $55 \%$ rather than $35 \%$, as was the case when only water was adopted, according to the report of Balakrishnan et al. [34]. These authors 
showed that, in addition to an increase in oxidized product yield, when the reaction is carried out in a water:ethanol mixture, extensive polymer cleavage also occurred. All these effects were rationalized by invoking the formation of more reactive hydroxyethyl radicals during the oxidation reaction [34]. Concerning spectroscopic characterization, the Raman spectrum in Figure 2 shows the characteristic peaks of NaAlg [35,36] where symmetrical and asymmetrical stretching vibrations of $-\mathrm{COO}^{-}$at 1420 and $1600 \mathrm{~cm}^{-1}$ occurred together with a $\mathrm{C}-\mathrm{O}$ stretch at $1330 \mathrm{~cm}^{-1}$ and a $\mathrm{C}-\mathrm{O}-\mathrm{C}$ stretch at $1100 \mathrm{~cm}^{-1}$.

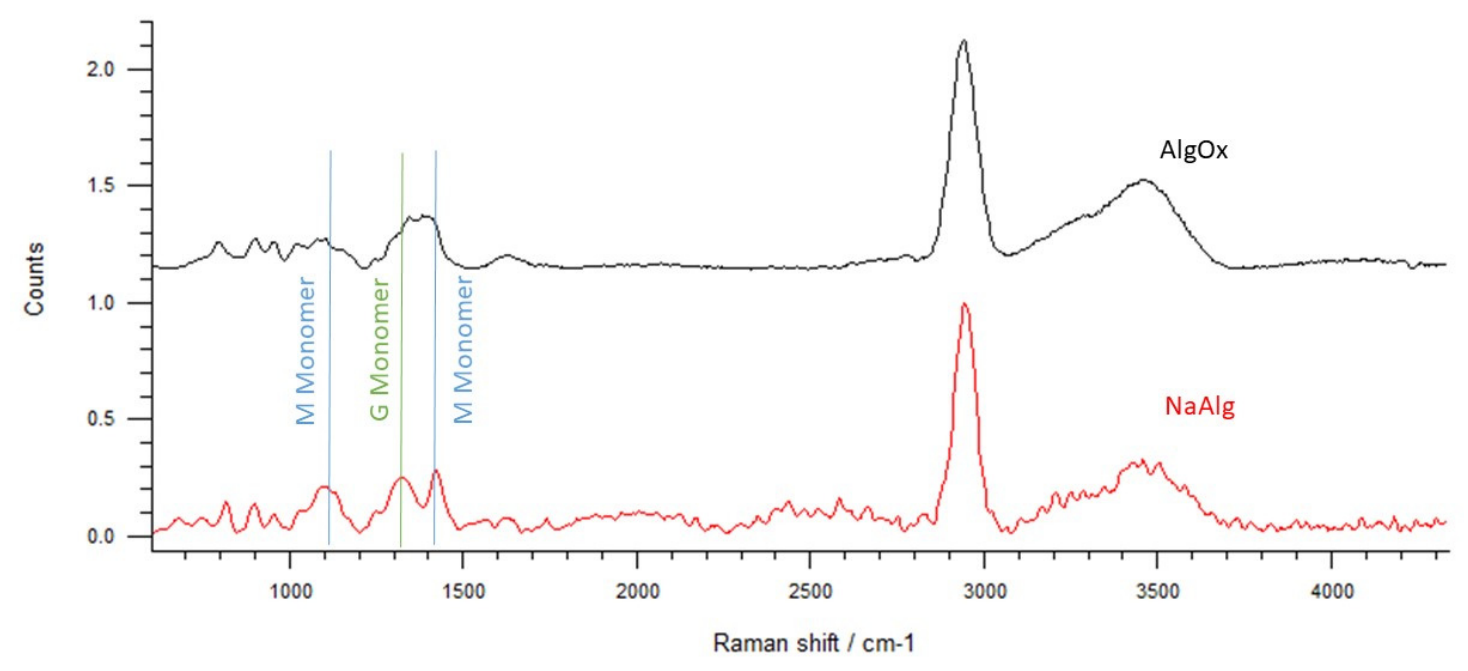

Figure 2. Raman spectra of NaAlg (red line) and AlgOX (black line).

When Raman spectra of NaAlg and AlgOX were compared (Figure 2), in the case of $\mathrm{AlgOX}$, the peaks corresponding to the ones of NaAlg were identified, together with a lowering of those at 1400 and $1050 \mathrm{~cm}^{-1}$, demonstrating the oxidation of the monomer M of the NaAlg backbone [34]; the peak at $1300 \mathrm{~cm}^{-1}$ indicated oxidation of the $\mathrm{G}$ monomer.

\subsection{SEM-EDX Investigations}

SEM microscopy is a useful method to analyze the morphology and formation of micro and nanostructures on a surface. In Figure $3 \mathrm{a}, \mathrm{b}$ NaAlg and AlgOX show similar external morphologies; from Figure $3 \mathrm{c}$ it can be deduced that the surface of AlgOX-DA seems thinner than those of NaAlg and AlgOX. Moreover, on the surfaces of AlgOX and AlgOX-DA, no micro- or nanostructures were detected. For NaAlg, AlgOX, and AlgOX-DA (Figure $4 \mathrm{a}-\mathrm{c}$ ), the recorded EDX spectra were combined with elemental analyses of the sample composition. The analyses of NaAlg and AlgOX (Figure 4a,b) showed that both samples had the same ratio of $\mathrm{O} / \mathrm{C}$, equal to 2, leading to the conclusion that $\mathrm{NaAlg}$ oxidation to AlgOX occurred. As a matter of fact, the oxidation from alcohol function to aldehyde only involves the loss of $\mathrm{H}$ atoms, so the ratio stays the same. Elemental analysis calculations of $\mathrm{AlgOX}$ (Figure $4 \mathrm{~b}$ ) led to achieving the following values for oxygen, carbon, and nitrogen: $52 \pm 2,33 \pm 3$ and $0.03 \pm 0.07$. For AlgOX-DA (Figure 4c) they were $45 \pm 4$, $46 \pm 6$, and $3 \pm 2$. The increase in the carbon percentage and the presence of nitrogen atoms were ascribable to DA, whereas the decrease of the oxygen atom content was due to the water loss that occurred when the imine bond was formed. Therefore, these findings confirm the formation of a Schiff base between AlgOX and DA. 
(a)

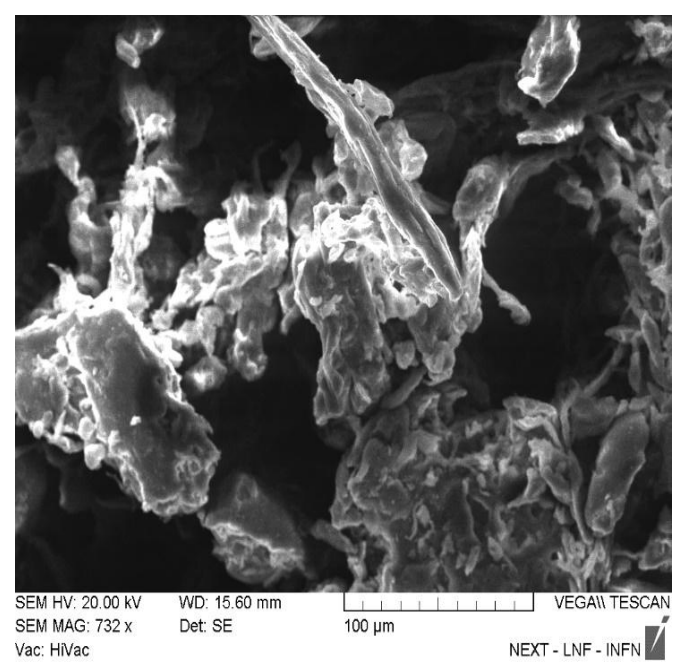

(b)

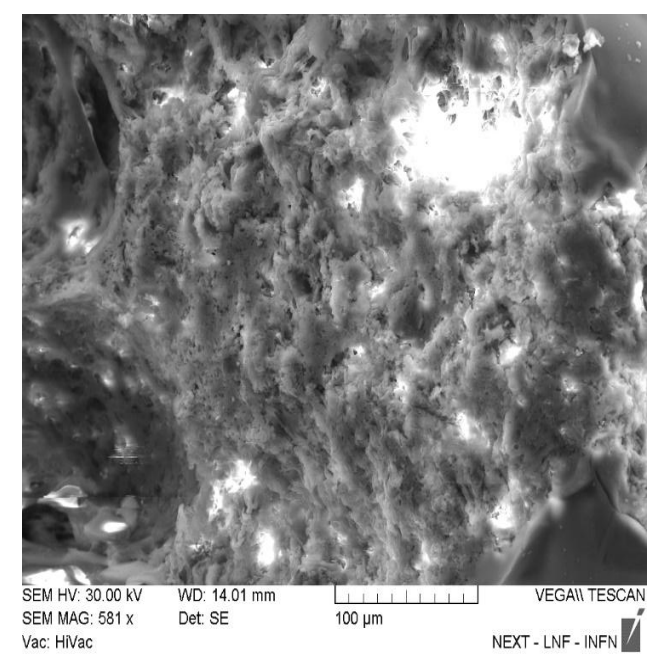

(c)

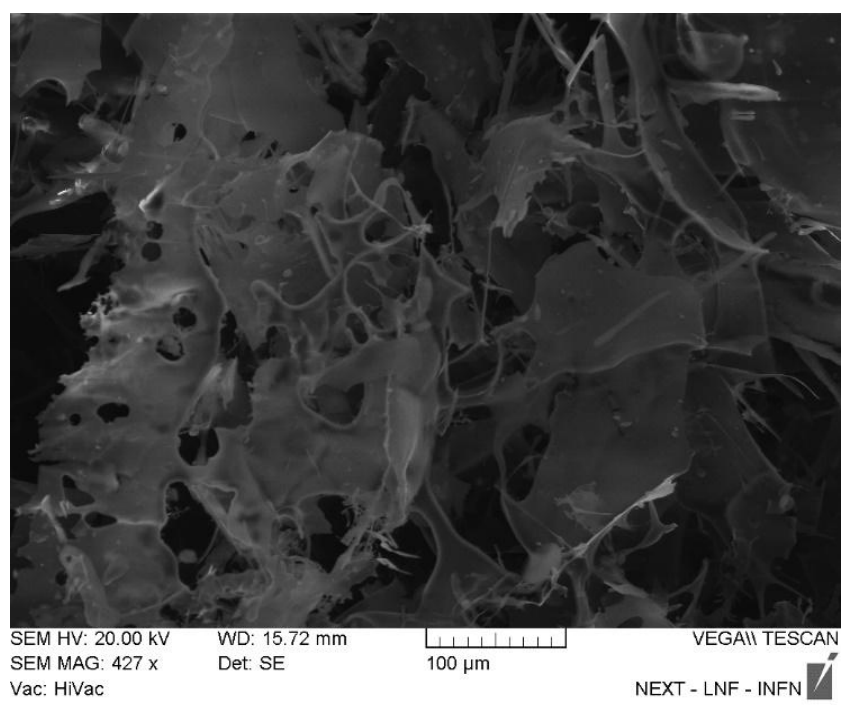

Figure 3. SEM images of (a) NaAlg; (b) AlgOX; and (c) AlgOX-DA.

\subsection{UV-Vis and FT-IR Analysis}

UV-vis spectra were used to gain information about the covalent linkage of DA to the NaAlg backbone. The UV-vis spectrum of the AlgOX-DA imine conjugate, as well as those of AlgOX and pure DA, are shown in Figure 5. ForAlgOX and AlgOX-DA UV-vis spectra, the initial part $(250 \mathrm{~nm})$ was too sharp and such effects seemed to be ascribable to the presence of water solvent. As can be seen, a characteristic peak appeared at $280 \mathrm{~nm}$, attributable to the absorbance of pure DA. In the case of the AlgOX-DA imine conjugate a shoulder was observed at $287 \mathrm{~nm}$, while neither the absorption peak nor the shoulder were detected in the corresponding spectrum of AlgOX. The shoulder at $287 \mathrm{~nm}$ may have been due to the successful linkage between AlgOX and DA, the absence of any peak at 320 and $425 \mathrm{~nm}$ also meant the absence of quinone and polydopamine structures, respectively [37]. 
(a)

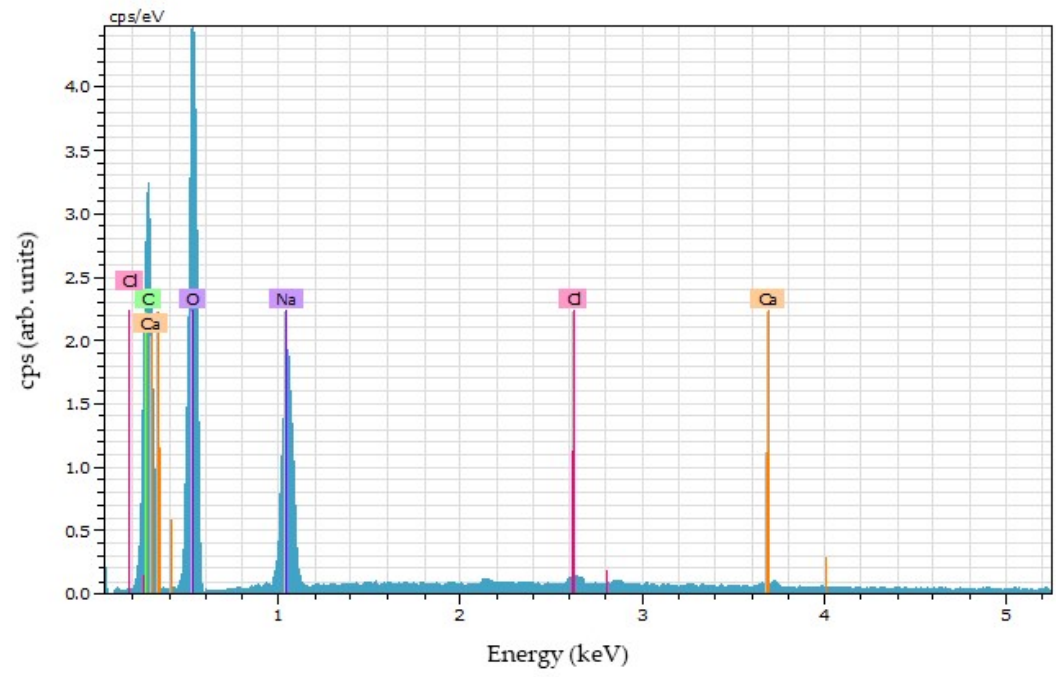

(b)

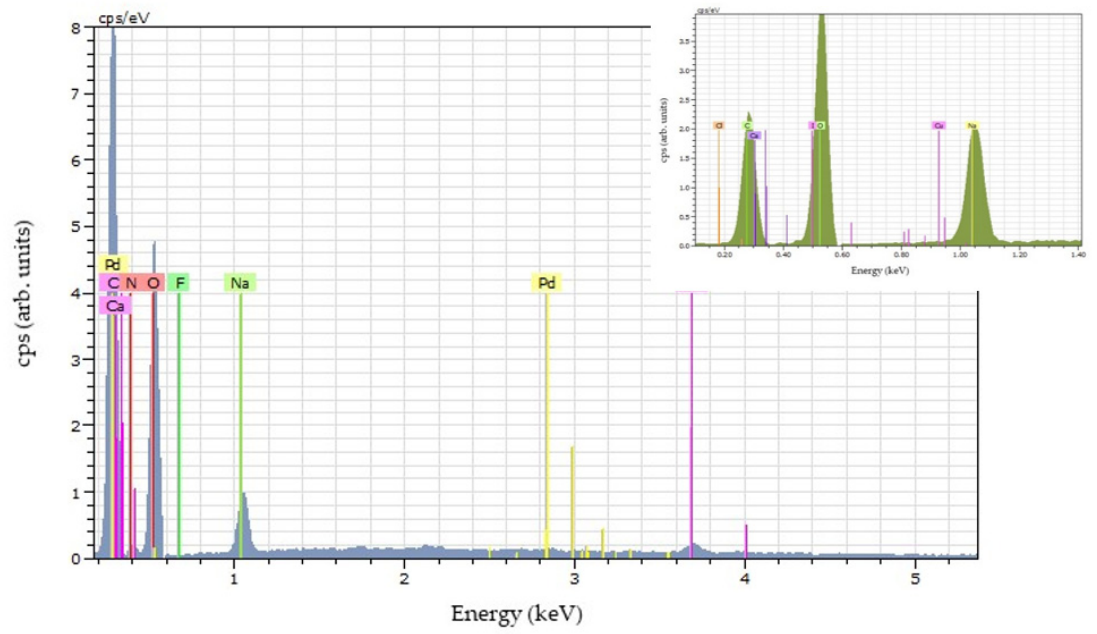

(c)

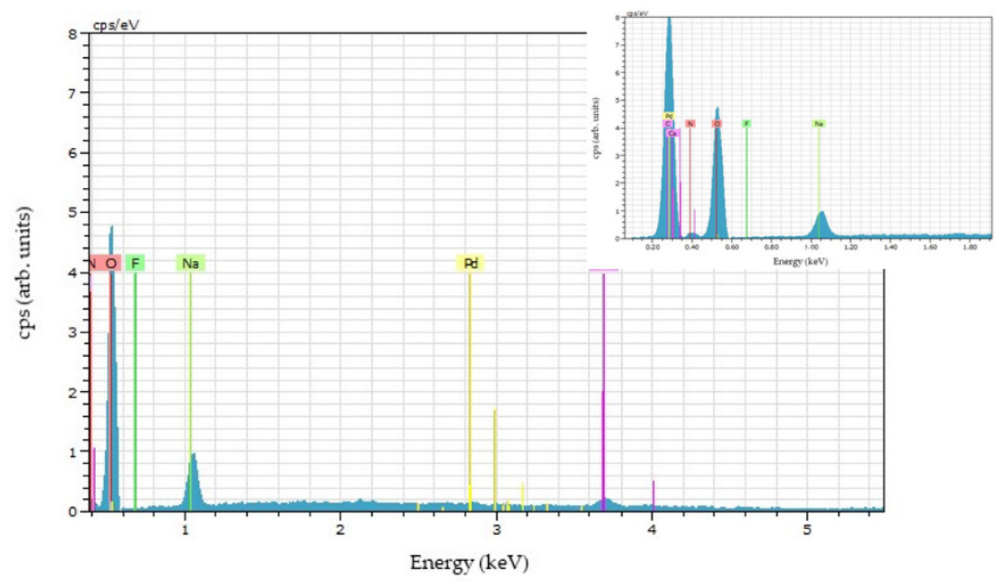

Figure 4. EDX spectra of $\mathrm{NaAlg}(\mathbf{a}) ; \mathrm{AlgOX}(\mathbf{b})$; and $\mathrm{AlgOX}-\mathrm{DA}(\mathbf{c})$. In the inset, the spectra is in the range of $0-2 \mathrm{keV}$. 


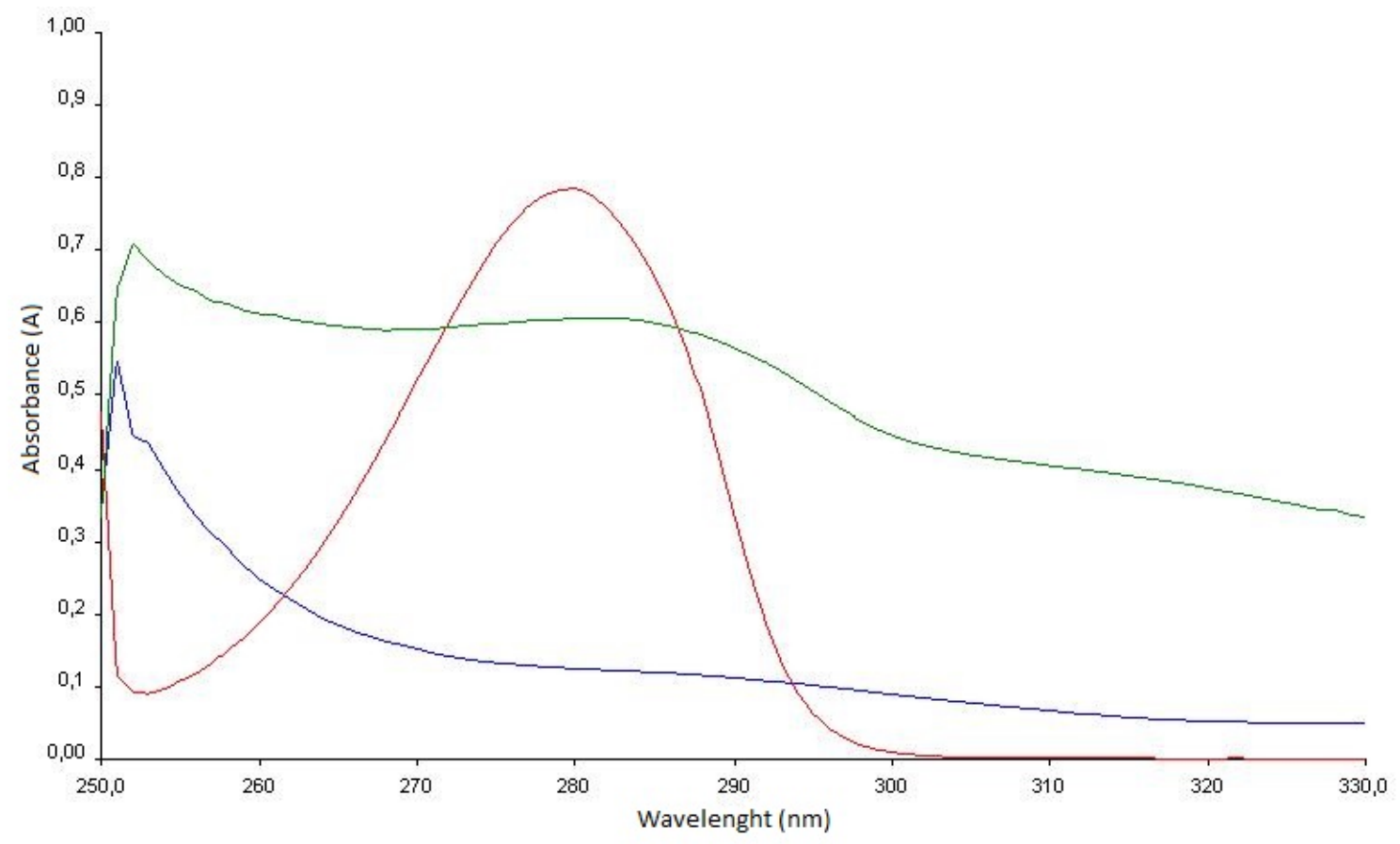

Figure 5. UV-spectra of pure DA (red); AlgOX (blue); and Alg OX-DA (green).

Concerning FT-IR spectra (Figure 6) for the DA spectrum, the strong and overlapped peaks between 1615 and $1584 \mathrm{~cm}^{-1}$ were ascribable to the bending of $\mathrm{N}-\mathrm{H}$ of the DA primary amine group and the stretching vibrations of $\mathrm{C}-\mathrm{C}$ belonging to the aromatic ring. Both spectra of AlgOX and AlgOX-DA showed a broad band at $3400 \mathrm{~cm}^{-1}$, ascribable to -OH groups of the polysaccharide vibrations and, in the case of FT-IR spectrum of AlgOX$\mathrm{DA}$, the $\mathrm{O}-\mathrm{H}$ group of the catechol ring should also be included in that band, as previously demonstrated by Yue et al. [37]. Moreover, in both FT-IR spectra, instead of the typical stretching of $C=O$ at $1700-1725 \mathrm{~cm}^{-1}$, the bands at $1622 \mathrm{~cm}^{-1}$ and at $1636 \mathrm{~cm}^{-1}$ were indicative of the symmetric stretching vibration for the carbonyl group for the aldehyde and the Schiff base groups, of AlgOX and AlgOX-Da, respectively.

\subsection{NMR Spectroscopy}

The signals at 5.3-5.2, 5.1-4.5 and 4.3-3.7 ppm were assigned to protons of mannuronic acid and guluronic acid units (Figure 7a) [26]. The oxidation of Alg to Alg OX was confirmed by the signal at $5.4 \mathrm{ppm}$ (Figure 7a), which corresponded to a hemiacetalic proton formed from aldehyde and the neighboring hydroxyl group, and by the absence of a signal at 9.5-10 ppm of a free aldehyde group, in agreement with Gomez [26].

The soluble macromolecules of AlgOX-DA were characterized in $\mathrm{D}_{2} \mathrm{O}$ solutions using ${ }^{1} \mathrm{H}-\mathrm{NMR}$ (see Gao [38]) in order to increase the solubility of AlgOX-DA in $\mathrm{D}_{2} \mathrm{O}$, the NMR tube was preheated at $40^{\circ} \mathrm{C}$.

The signals of Figure $7 \mathrm{~b}$, located at $6.77(1 \mathrm{H}, \mathrm{d}, J 7.8 \mathrm{~Hz}, \mathrm{H}-1$ in Figure $7 \mathrm{~b}), 6.71(1 \mathrm{H}$, d, J $1.8 \mathrm{~Hz}), 6.63(1 \mathrm{H}, \mathrm{dd}, J 17.8 \mathrm{~Hz}, J 21.8 \mathrm{~Hz}), 3.10(2 \mathrm{H}, \mathrm{t}, J 7.8 \mathrm{~Hz})$, and $2.74(2 \mathrm{H}, \mathrm{t}$, $J 7.8 \mathrm{~Hz}$ ) ppm correspond to DA protons. In the ${ }^{1} \mathrm{H}-\mathrm{NMR}$ spectra of AlgOX-DA (Figure 7b) additional peaks appeared. In ${ }^{1} \mathrm{H}-\mathrm{NMR}$ spectrum of AlgOX-DA (Figure $7 \mathrm{~b}$ ) additional peaks appeared, with respect to the ${ }^{1} \mathrm{H}$-NMR spectrum of AlgOX (Figure 7a), deriving from the protons in aromatic ring of the bonded DA $(6.6 \sim 6.8 \mathrm{ppm})$ and from the imminic proton ( $\mathrm{HC}=\mathrm{N} 7.5 \mathrm{ppm})$.

Comparing the integral of $\mathrm{HC}=\mathrm{N}(7.5 \mathrm{ppm})$ with that of the $H-1$ unreacted DA signal located at $6.77 \mathrm{ppm}$, the percentage of incorporated DA in AlgOX-DA was $0.11 \%$, which corresponded to $0.04 \mathrm{mmol}$ of neurotransmitter. Working with $0.127 \mathrm{mmol}$ of initial monomer-type units of $\mathrm{AlgOX}$, the determination of the resulting degree of substitution (DS) was $31 \%$, as determined by ${ }^{1} \mathrm{H}-\mathrm{NMR}$ spectroscopy. The amount of DA covalently 
bound to the AlgOX-DA imine conjugate was also determined by hydrolysis under strong acidic conditions, as described in Section 2.6, and it was found to be equal to $36 \mu \mathrm{g}$ of $\mathrm{DA} / \mathrm{mg}$ of AlgOX-DA imine conjugate.

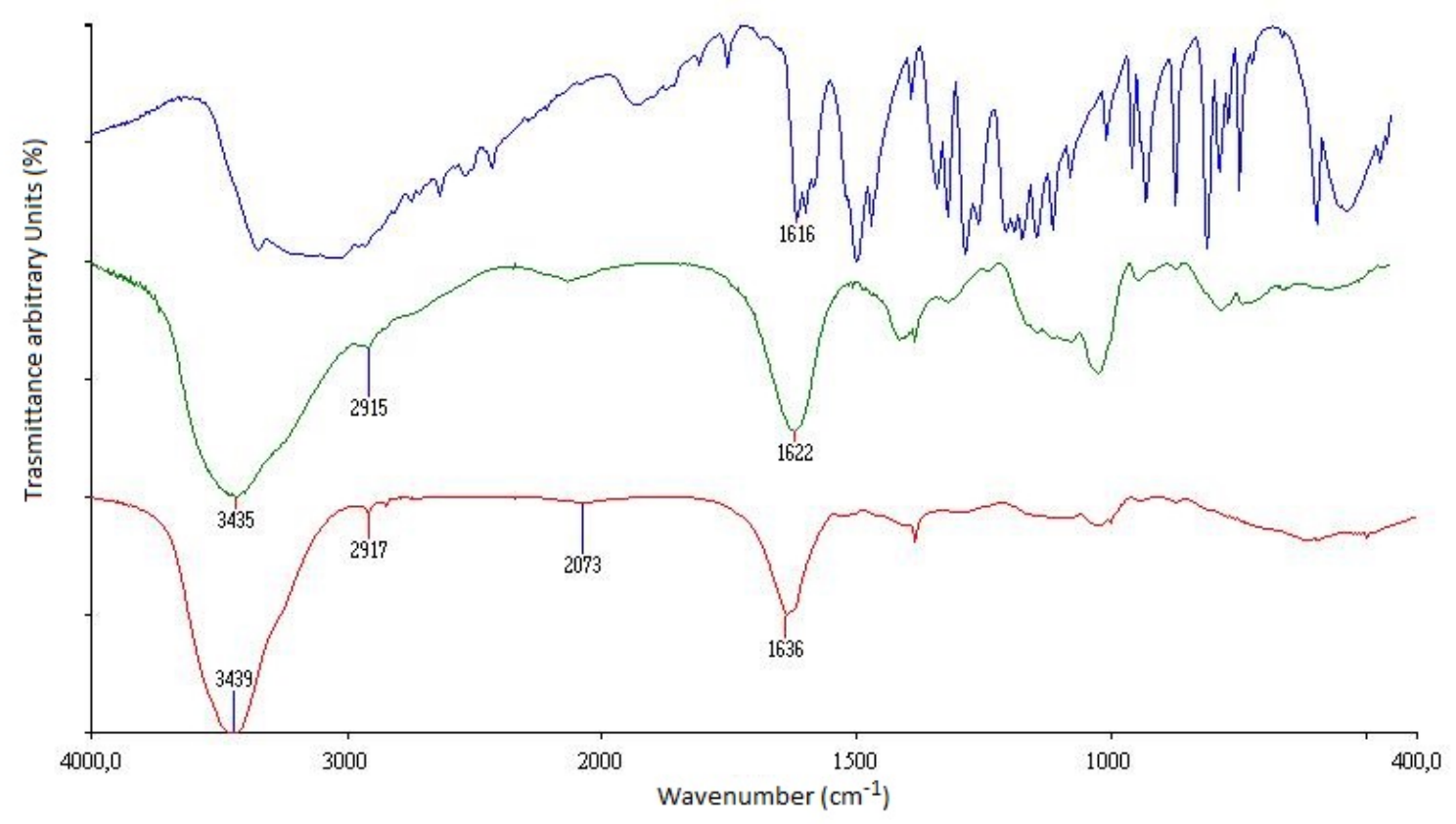

Figure 6. FT-IR spectra of pure DA (blue); pure Alg OX (green); and Alg OX-DA (pink). As can be seen, in the spectrum of $\mathrm{AlgOX}$, the band corresponding to the aldehyde vibration was not detected.

\subsection{Thermal Analysis via DSC}

Prior to performing DSC analysis, melting point values were acquired for AlgOX and AlgOX-DA. Precisely, AlgOX started to decompose at temperatures higher than $200{ }^{\circ} \mathrm{C}$ and, at temperatures higher than $250^{\circ} \mathrm{C}$, decomposition also started for AlgOX-DA. Information on the solid state of AlgOX-DA was obtained by comparing its DSC thermogram with those of AlgOX and pure DA (Figure 8). The DSC thermogram of DA is characterized by a sharp endothermic peak at $250^{\circ} \mathrm{C}$, corresponding to the its melting point. The DSC curve of the AlgOX-DA imine conjugate also showed an endothermic peak at $130{ }^{\circ} \mathrm{C}$, even though it was less intense, probably due to the loss of the physically bound water on the polysaccharide derivative. The thermogram of AlgOX showed an endothermic peak at $138^{\circ} \mathrm{C}$ plus two more peaks at $127^{\circ} \mathrm{C}$ and $162{ }^{\circ} \mathrm{C}$. These last peaks, following literature suggestions, should be due to the loss of water associated with the hydrophilic groups of $\mathrm{NaAlg}$ [39]. In the case of the peak at $128^{\circ} \mathrm{C}$, such a temperature value was the end set of an endothermic peak, ascribable to the backbone of non-oxidized NaAlg [39].

\subsection{XPS Studies}

X-ray photoelectron spectroscopy analyses were performed to gain insight into the surface chemical composition of the novel AlgOX-DA conjugate. DA and AlgOX were also measured, being starting materials and useful for controls. XPS elemental percentages are reported in Table 1, while carbon and nitrogen high resolution spectral regions are depicted in Figure 9. 
Table 1. XPS surface elemental percentages for DA, AlgOX, and AlgOX-DA Schiff base. The values are indicated as mean values averaged from at least three replicates; the errors are expressed as standard deviation $\pm 1 \mathrm{~S}$.

\begin{tabular}{ccccccc}
\hline Sample & \%C & \%N & \%O & \%Cl & \% Na & \%I \\
\hline DA & $67.1 \pm 0.9$ & $6.6 \pm 0.5$ & $22.0 \pm 0.9$ & $4.4 \pm 0.5$ & - & - \\
AlgOX & $46 \pm 3$ & $0.4 \pm 0.5$ & $41.7 \pm 1.8$ & - & $9.2 \pm 1.0$ & $2.3 \pm 0.5$ \\
AlgOX-DA & $69.3 \pm 1.6$ & $4.6 \pm 1.0$ & $25.6 \pm 0.5$ & - & $0.5 \pm 0.5$ & $0.1 \pm 0.2$ \\
\hline
\end{tabular}

(a)

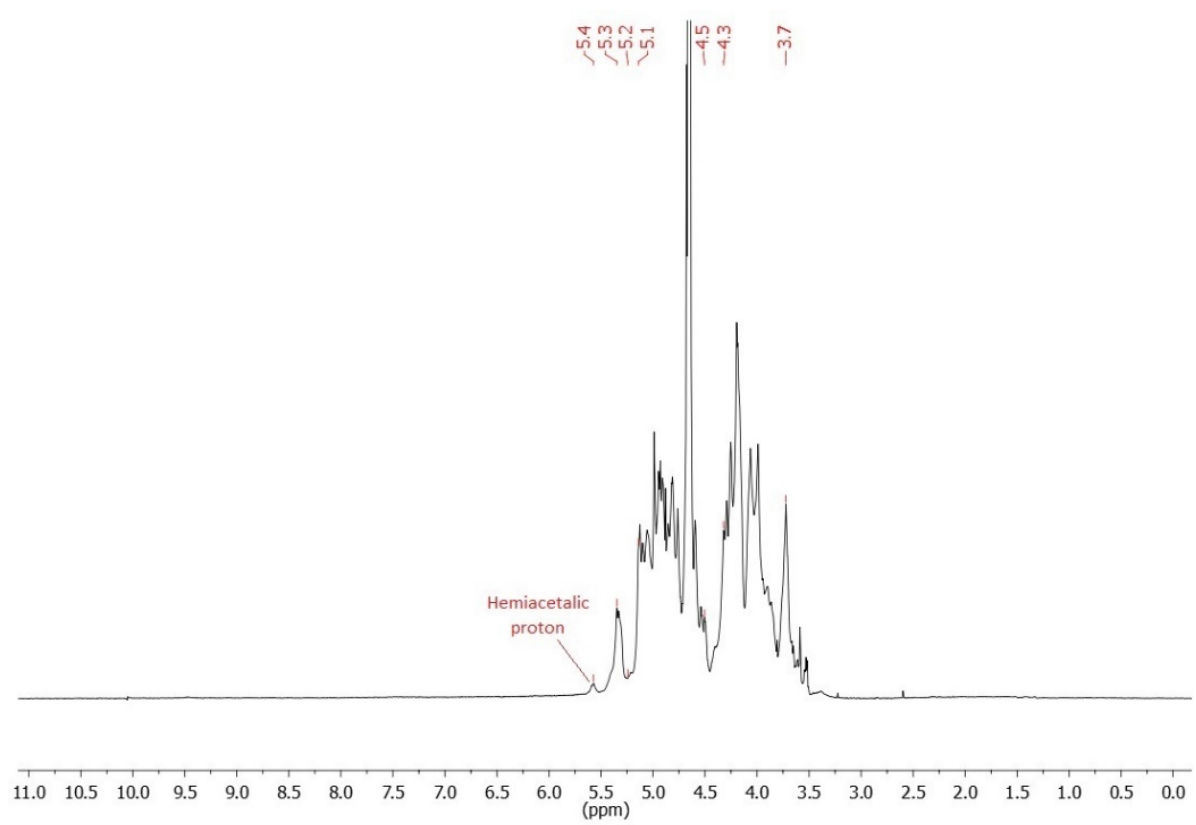

(b)

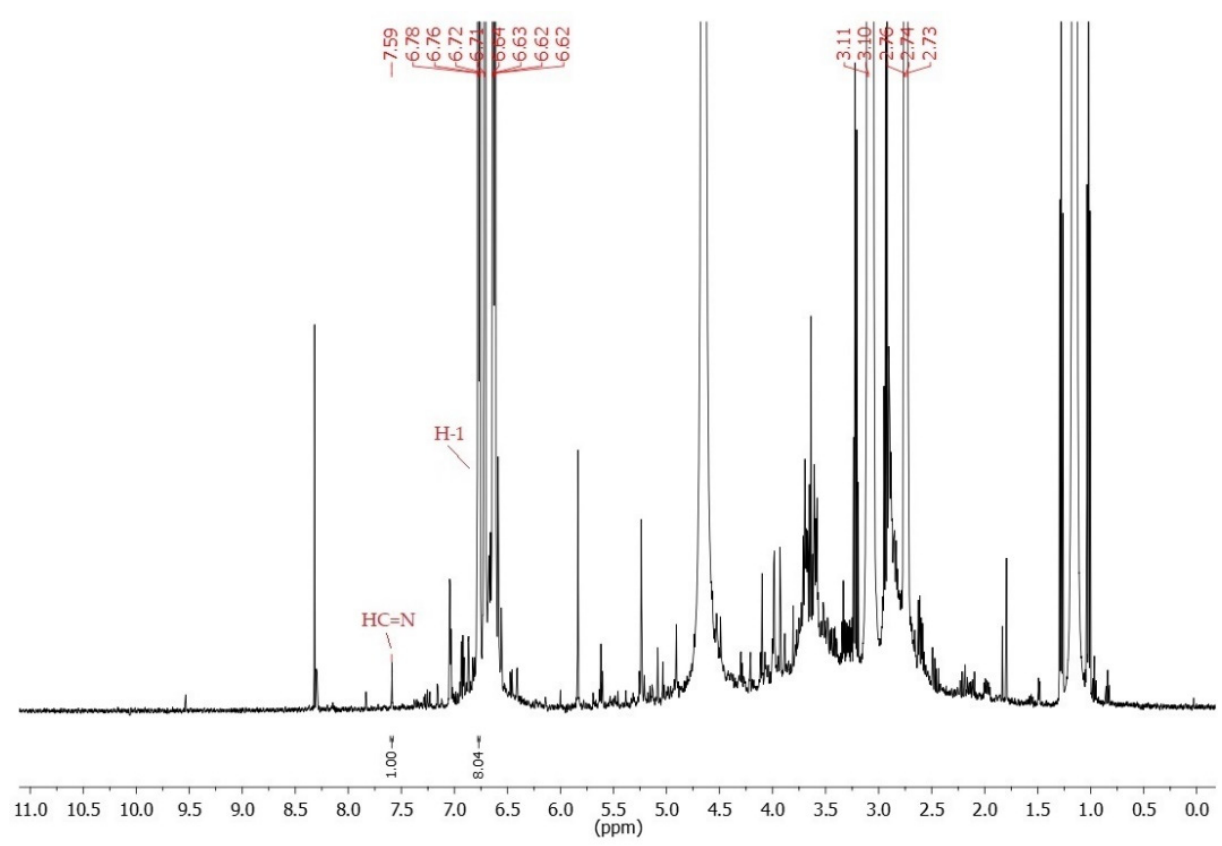

Figure 7. (a) ${ }^{1} \mathrm{H}$ NMR spectrum of $\mathrm{AlgOX}$ in $\mathrm{D}_{2} \mathrm{O}$ at $40{ }^{\circ} \mathrm{C}$ with identification of the main signals; (b) ${ }^{1} \mathrm{H}$ NMR spectrum of AlgOX-DA in $\mathrm{D}_{2} \mathrm{O}$ at $40{ }^{\circ} \mathrm{C}$ with identification of the main signals. 

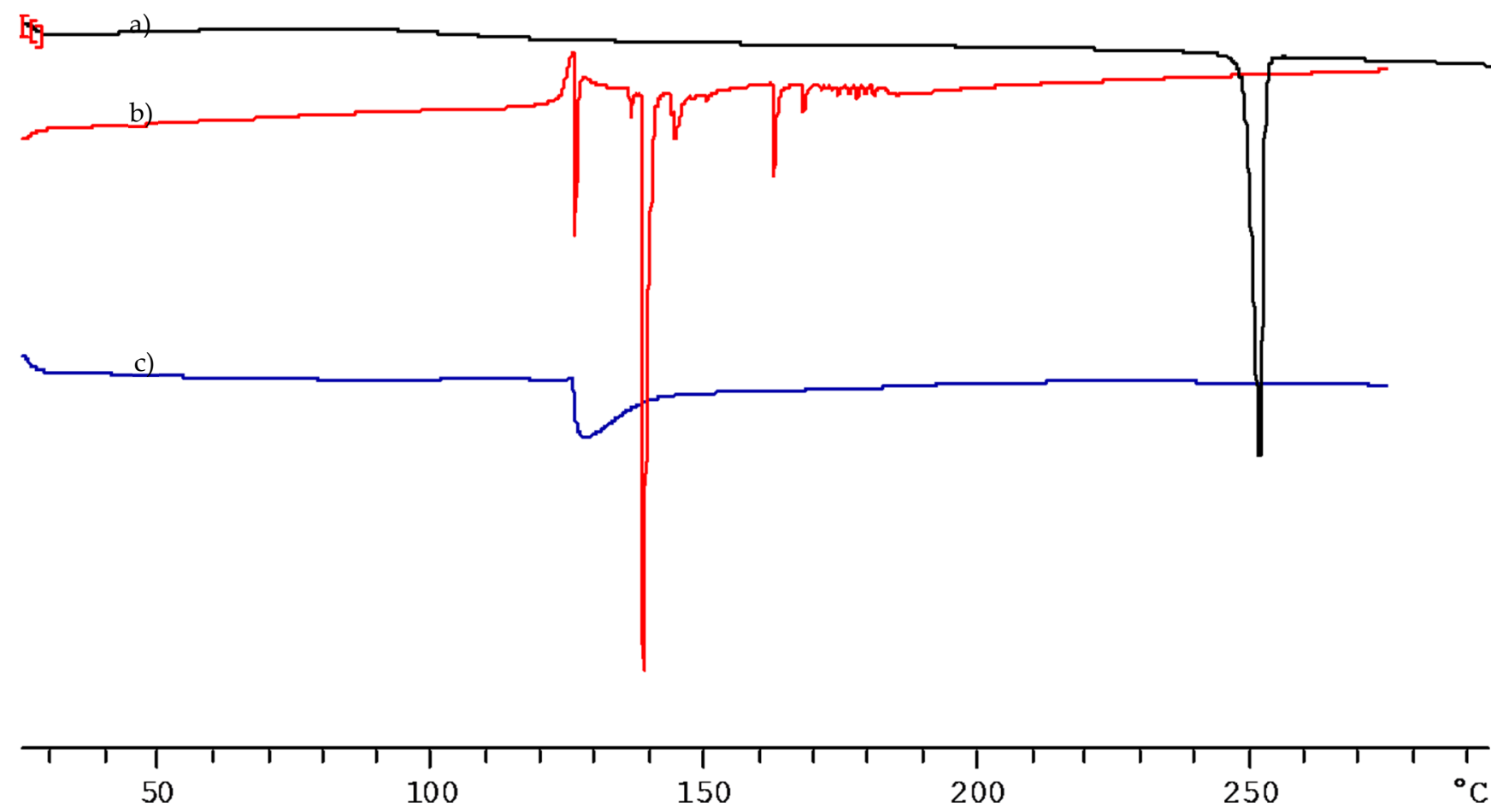

Figure 8. DSC thermograms of pure DA (a); pure AlgOX (b); and AlgOX-DA (c).
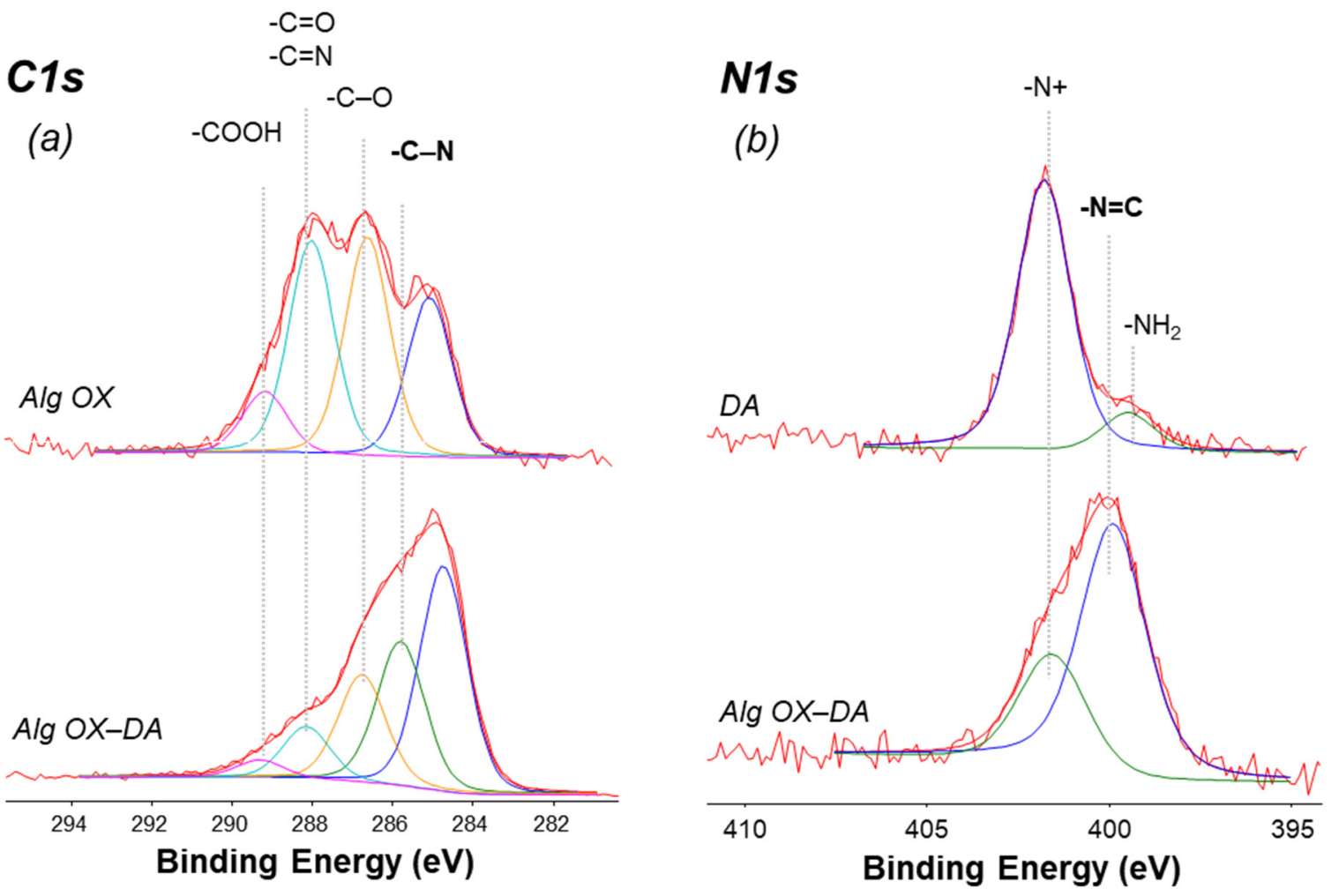

Figure 9. (a) C1s curve fitted XP spectrum of AlgOX and AlgOX-DA imine conjugate; (b) N1s curve fitted XP spectrum of DA and AlgOX-DA imine conjugate. Peak component attribution is reported for all the spectral regions.

As expected, carbon, oxygen, and nitrogen were detected on the surface of pure DA, along with chlorine due to the hydrochloride form of dopamine. The surface of 
AlgOX showed residual traces of the $\mathrm{NaIO}_{4}$ used in the oxidation protocol of alginate. Nevertheless, the AlgOX-DA showed a good amount of surface nitrogen, demonstrating the successful preparation of the conjugate. This was further confirmed by detailed C1s and N1s investigations. Figure 9a shows a comparison of curve-fitted C1s of AlgOX and AlgOX$\mathrm{DA}$, with the attribution of the carbon chemical environments. A similar evaluation is presented in Figure 9b, where the curve-fitted N1s of AlgOX-DA is compared with the N1s of DA. In both cases, the spectra of the conjugate presented peak components, univocally assignable to the formation of the Schiff base; that is, the $-\mathrm{C}-\mathrm{N}$ peak at $285.7 \pm 0.1 \mathrm{eV}$ in $\mathrm{C} 1 \mathrm{~s}$ spectrum, and the $-\mathrm{N}=\mathrm{C}$ at $400.1 \pm 0.1 \mathrm{eV}$ in $\mathrm{N} 1 \mathrm{~s}$ region.

\subsection{Mucoadhesion Studies}

Among the different methods for in vitro evaluation of mucoadhesive effects, herein, the changes in transmittance values of mucin upon contact with AlgOX and AlgOX-DA were monitored in order to assess the rank order of the mucoadhesive materials [26]. As shown in Figure 10, from the turbidimetric measurements, the mucoadhesive properties of the tested materials could be classified in the following rank order: AlgOX-DA $>$ AlgOX $>$ HEC.

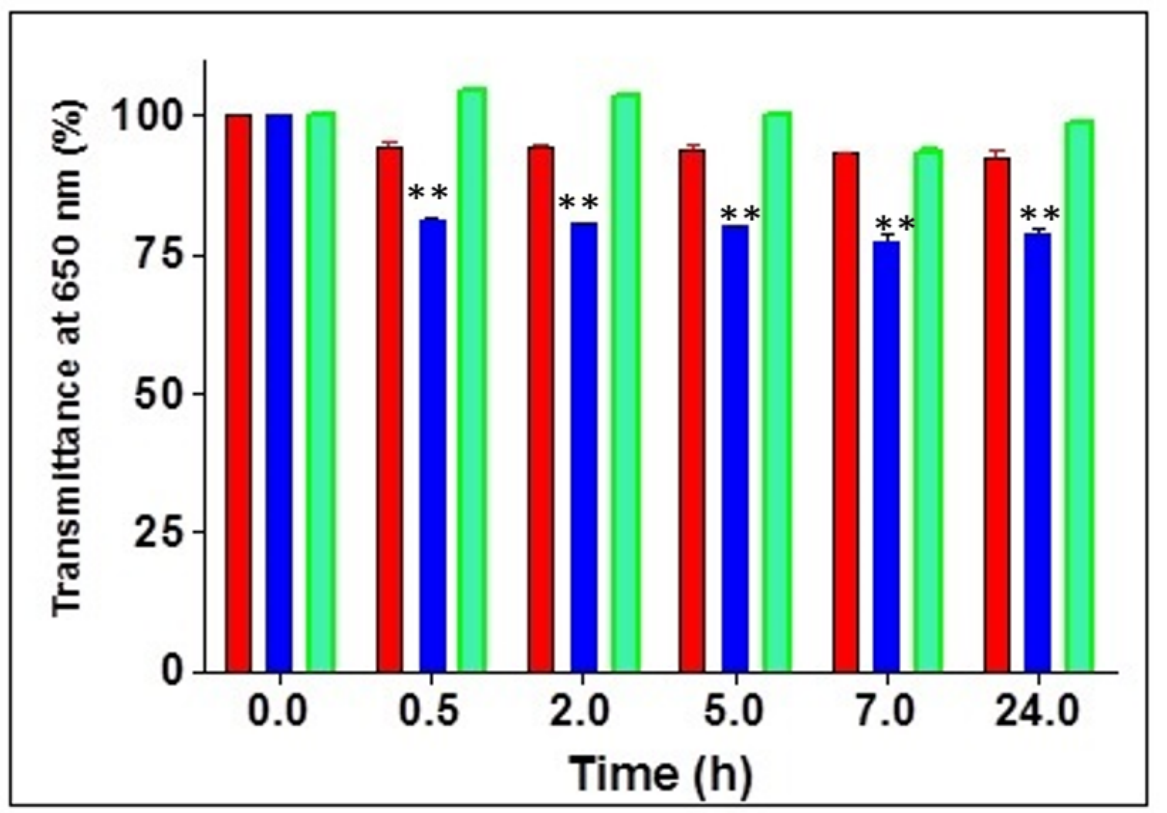

Figure 10. Mucoadhesive effects in SNF of AlgOX (red bars) and AlgOX-DA imine conjugate (blue bars). HEC (green bars) was used as positive control; ** $p \leq 0.001$.

Although HEC was already employed as a reference semisynthetic polymer due to its good mucoadhesive features [40], from $30 \mathrm{~min}$ to $24 \mathrm{~h}$, the transmittance values detected at the wavelength of $650 \mathrm{~nm}$ for Alg OX-DA were characterized by a statistically very significant difference $(p<0.01)$ vs. the control HEC (Figure 10). From turbidimetric measurements, the decrease in transmittance vs. HEC after $24 \mathrm{~h}$ of incubation resulted in being greater in the case of AlgOX-DA imine conjugate than for AlgOX. The corresponding differences were evaluated using the ANOVA approach and both Bonferroni's and Tukey's post hoc tests. It was found that the difference between AlgOX vs. HEC was not statistically significant $(p>0.05)$ while the difference of AlgOX vs. AlgOX-DA imine conjugate was significant $(p<0.01)$ as was that of AlgOX-DA imine conjugate vs. HEC, with $p<0.01$.

\subsection{In Vitro DA Release from AlgOX-DA in SNF and PBS}

The behavior of AlgOX-DA to the chemical hydrolysis in the release the neurotransmitter was evaluated under two different $\mathrm{pH}$ media. In Figure 11a, the release profile of AlgOX-DA was determined in simulated nasal electrolyte solution without enzymes buffered at $\mathrm{pH} 6$, where $13 \%$ of DA was delivered in $24 \mathrm{~h}$, according to a sustained re- 
lease trend. Precisely, the receiving medium changed color from clear to pale yellow in the frame time of $3 \mathrm{~h}$ to $24 \mathrm{~h}$; perhaps in good agreement with progressive Schiff base hydrolysis. According to the literature, it has already been seen that, under the conditions used ( $\mathrm{pH} 6.0$ without enzymes), further oxidative products of the neurotransmitter DA are almost absent [41]. The diffusion from a kinetic point of view may be described by the Higuchi equation (i.e., $C_{T}=K_{H} \times t_{1 / 2}$, where $C_{T}$ is the amount of DA at time $t$ ) and the degradation reactions, which may be expressed by a first order reaction rate. The resulting global reaction rate can be simplified to the Higuchi equation only for very short time or for very slow degradation reactions. Following this approach, by plotting the percentages of cumulative DA release against the square root of the time, the Higuchi parameter $\left(\mathrm{K}_{\mathrm{H}}\right)$ can be obtained. When PBS was adopted as the receiving medium, then autoxidation of DA due to the higher $\mathrm{pH}$ value is of aid to explain the percentage reduction of DA delivered from $5 \mathrm{~h}$ (Figure 11c), reaching around $5 \%$ of neurotransmitter in the medium at $24 \mathrm{~h}$. In the case of the AlgOX-DA imine conjugate in SNF, a KH value of $7.476 \pm 1.828$ (\% of DA released $\times \mathrm{h}^{-1 / 2}$ ) was calculated for the first $3 \mathrm{~h}$ of release. On the other hand, for the first $3 \mathrm{~h}$ of release in PBS ( $\mathrm{pH}$ 7.4) the calculated $\mathrm{K}_{\mathrm{H}}$ value was $11.90 \pm 4.563(\%$ of DA released $\times \mathrm{h}^{-1 / 2}$ ). From these results, it appears that, under acidic conditions (i.e., SNF), a sustained neurotransmitter release occurs while, in neutral/alkaline conditions (i.e., PBS), a faster release was observed.
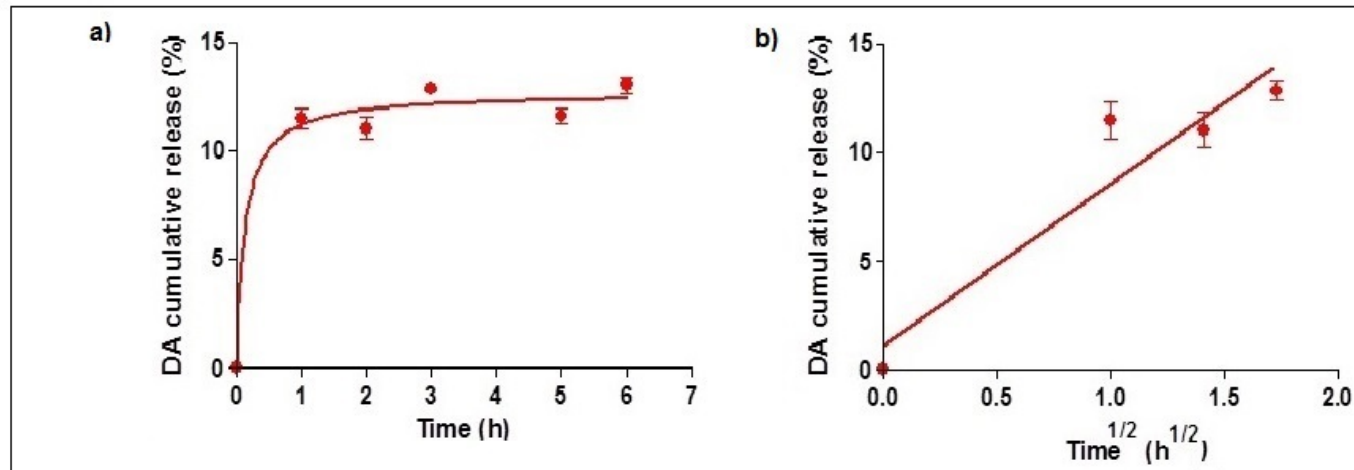

c)

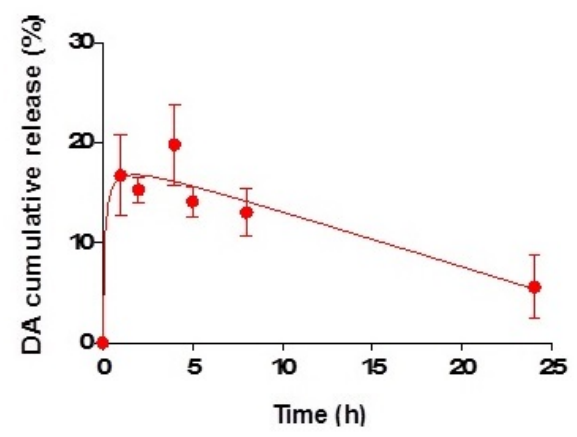

d)

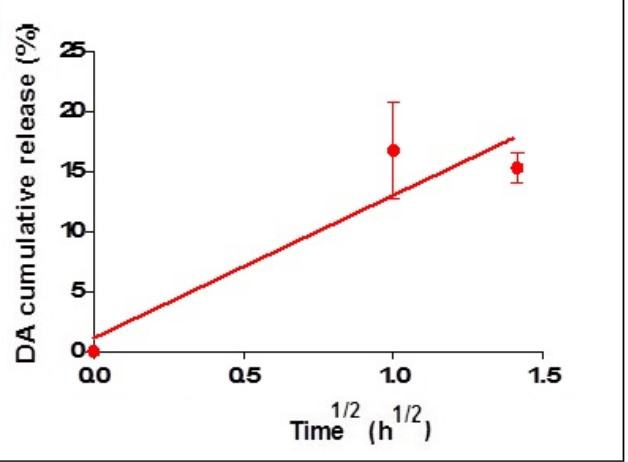

Figure 11. (a) DA cumulative release in SNF from AlgOX-DA imine conjugate; (b) square root dependency of DA released in SNF from AlgOX-DA imine conjugate; (c) DA cumulative release in PBS ( $\mathrm{pH}=7.4)$ from AlgOX-DA imine conjugate; (d) square root dependency of DA released in PBS ( $\mathrm{pH}=7.4)$ from AlgOX-DA imine conjugate.

\subsection{FITC Labeling of AlgOX}

The labeling procedure described for AlgOX was similar to that followed for some chitosan derivatives, such as fluorescent carboxymethylchitosan already preparedby us [28] and fluorescent glycol chitosan [31]. The resulting fluorescent AlgOX was obtained as an orange powder with a labeling efficiency of $2.8 \mu \mathrm{g}$ FITC/mg FITC-AlgOX conjugate with a yield of the final fluorescent biomaterial equal to $27 \% \pm 3$. 


\subsection{PCS Analysis and SNOM Visualization}

According to PCS methodology, the particle sizes of AlgOX and AlgOX-DA were determined (Table 2). Interestingly, the mean diameter of the imine conjugate AlgOX-DA was lower than that of AlgOX, although the polydispersity index, which is correlated to the colloid distribution, was similar and slightly high for both materials (Table 2). Furthermore, visualization of fluorescent AlgOX was also performed via SNOM microscopy. Figure $12 \mathrm{~b}$ shows a fluorescence SNOM image with the corresponding shear-force topography (Figure 12a) of an FITC-AlgOX sample at a concentration of $1 \mathrm{mg} / \mathrm{mL}$, taken as such. In the SNOM microphotographs, lighter areas are typical of a stronger emission. The shear-force image shows microstructures with a width of a few microns and a height of several tens of nm (Table 3). Similar topographic images were observed through all samples and may be considered representative of AlgOX structure [42]. The fluorescence spectroscopic SNOM images show emission distribution in areas that corresponds to the microstructures and the largest emission is always observed around the FITC-AlgOX microstructures. From these observations it is possible to state that there is almost a complete one-to-one correspondence between topographical microstructures and maxima in fluorescence emission, indicating that most of the fluorophore was stuck to AlgOX backbone, evidencing uniform fluorescence distribution throughout the sample. Additionally, in a few areas in Figure 12a,b, a fluorescence emission is shown, even though no topographical microstructures were observed. A similar fluorescence behavior was observed when FITCAlgOX was processed at a concentration of $0.01 \mathrm{mg} / \mathrm{mL}$ (Figure 12c,d), even though it was very difficult to find appropriate areas where a microstructure gave raise to fluorescence signal, due, perhaps, to the high level of dilution of the fluorescent probe. A statistical analysis of FITC-AlgOX $(1 \mathrm{mg} / \mathrm{mL})$ characteristics according to AFM-SNOM observations is summarized in Table 3.

Table 2. Mean particle size of AlgOX and AlgOX-DA according to PCS analysis.

\begin{tabular}{ccc}
\hline Sample & \multicolumn{2}{c}{ Mean Diameter Polidispersity Index } \\
\hline AlgOX & $233(\mathrm{SD}=4)$ & $0.36(\mathrm{SD}=0.02)$ \\
AlgOX-DA & $164(\mathrm{SD}=21)$ & $0.39(\mathrm{SD}=0.01)$ \\
\hline
\end{tabular}

Table 3. Statistical analysis of FITC-AlgOX $(1 \mathrm{mg} / \mathrm{mL})$ properties according to SNOM observations.

\begin{tabular}{|c|c|c|c|c|c|}
\hline \multirow{2}{*}{$\begin{array}{c}\text { Sample } \\
-\end{array}$} & \multicolumn{3}{|c|}{ Topography } & \multicolumn{2}{|c|}{ Fluorescence } \\
\hline & $\begin{array}{l}\text { Mean Roughness } \\
\text { RMS (nm) }\end{array}$ & Mean Heights (nm) & Mean Areas $\left(\mu \mathrm{m}^{2}\right)$ & $\begin{array}{l}\text { Mean Areas } \\
\text { Spot (a.u.) }\end{array}$ & Mean Intensity (a.u.) \\
\hline FITC Alg OX & $58.25(\mathrm{SD}=36.37)$ & $198.06(\mathrm{SD}=122.82)$ & $59.08(\mathrm{SD}=78.05)$ & $72.60(\mathrm{SD}=80.91)$ & $48.13(\mathrm{SD}=62.11)$ \\
\hline
\end{tabular}

\subsection{Cell Viability}

In this study, we confirmed that DA reduces the viability of SH-SY5Y cells in a dosedependent $(1-50 \mu \mathrm{M})$ manner (Figure 13a), but such cytotoxic effect decreased when DA was linked to AlgOX in the imine conjugate AlgOX-DA (Figure 13b). Notably, AlgOX completely reversed the effect on cell vitality of $10 \mu \mathrm{g} / \mathrm{mL}$ DA, although at concentrations above $100 \mu \mathrm{g} / \mathrm{mL}$ a significative reduction in cell viability was observed (Figure 13a). Moreover, FITC-AlgOX was incubated with SH-SY5Y for $24 \mathrm{~h}$, and at any tested concentration no effect on SH-SY5Y cell viability was observed. 

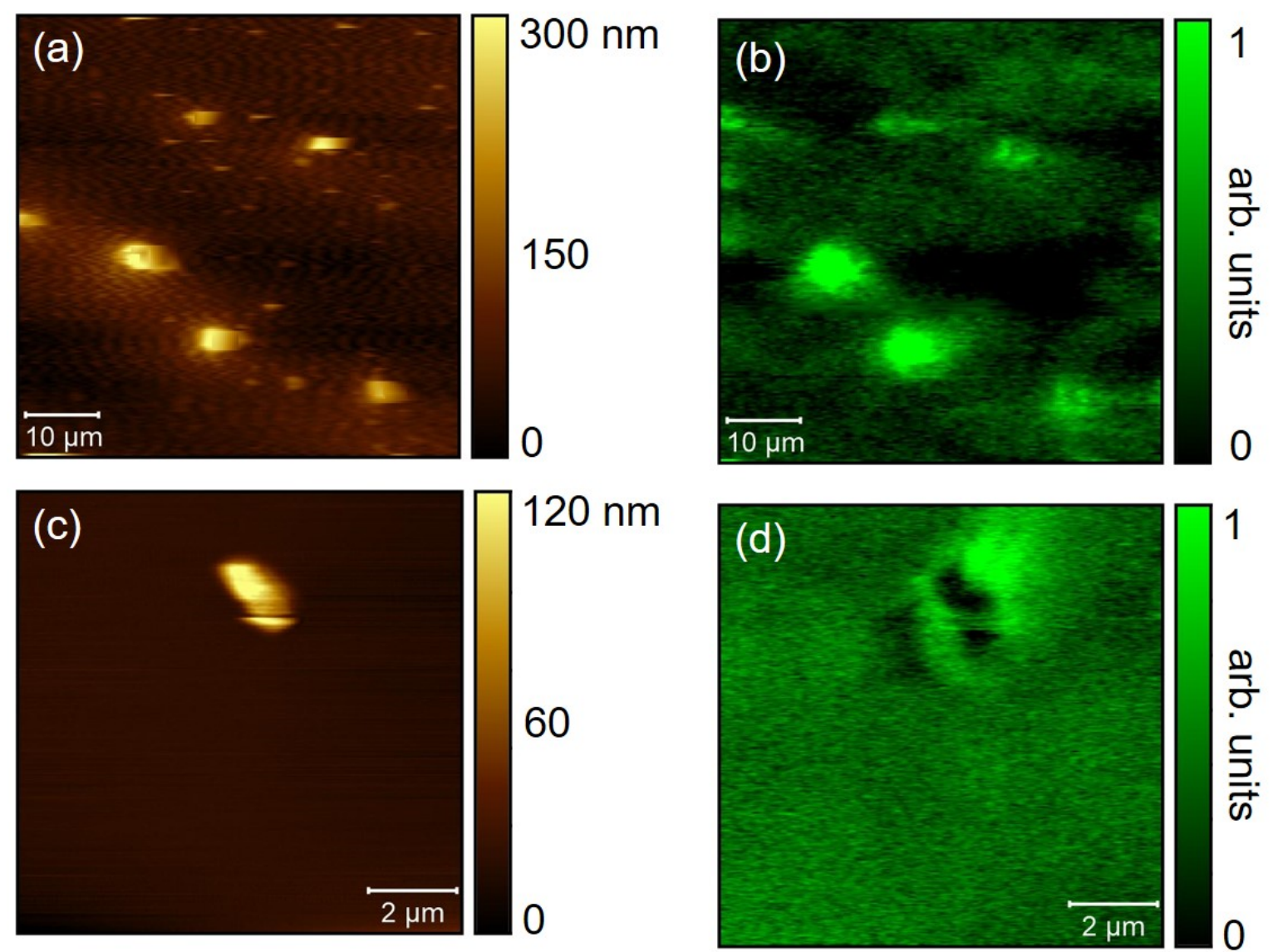

Figure 12. SNOM topography (left) and fluorescence (right) images of FITC-AlgOX $(1 \mathrm{mg} / \mathrm{mL})$ deposited on glass via micropipetting. (a) and (b) refer to FITC-AlgOX concentration of $1 \mathrm{mg} / \mathrm{mL}$; (c) and (d) refer to FITC-AlgOX concentration of $0.01 \mathrm{mg} / \mathrm{mL}$.

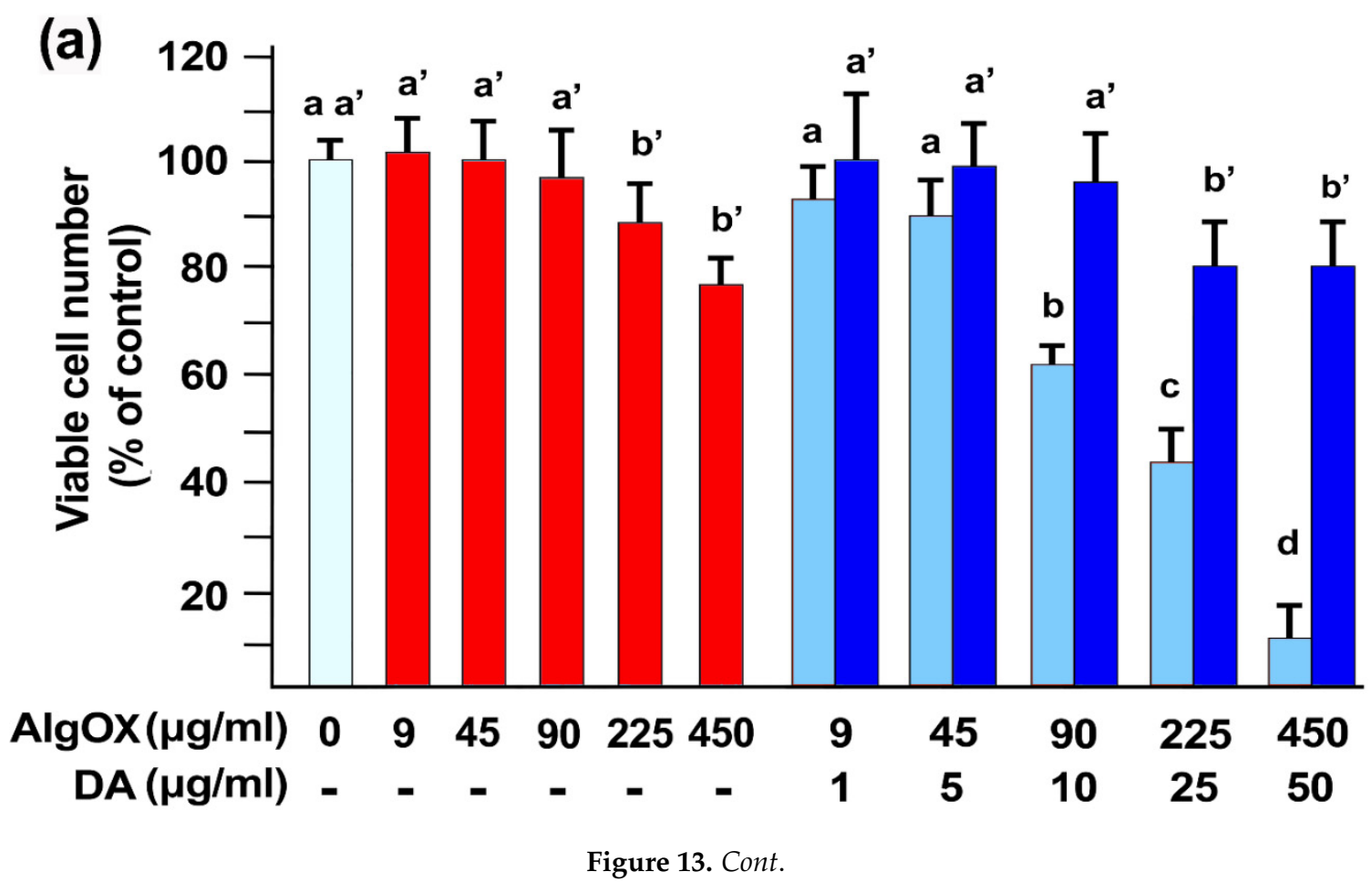




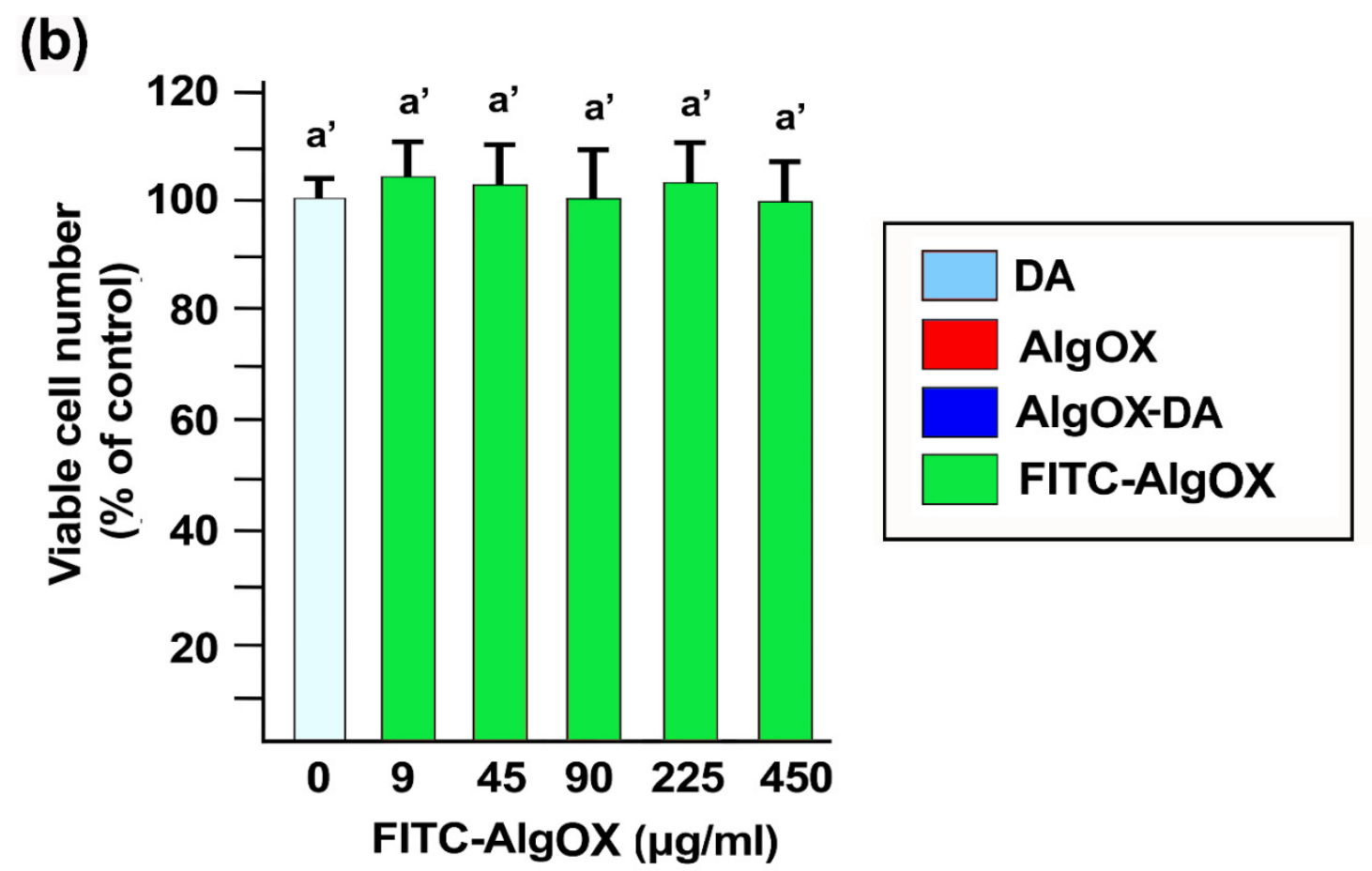

Figure 13. Sensitivity of SH-SY5Y cells to AlgOX and FITC-AlgOX. Cells were treated by varying concentrations of DA, AlgOX, AlgOX-DA (a) or FITC-AlgOX (b) and viable cell numbers were determined $24 \mathrm{~h}$ later with sulforhodamine B (SRB) assay. The data are means \pm S.D. of four different experiments run in eight replicates and are presented as percent of control. Values of the histograms with shared letters are not significantly different according to Bonferroni/Dunn's post hoc tests.

\section{Discussion}

The aim of the present work was to synthesize and characterize a novel macromolecular AlgOX-DA imine conjugate and to evaluate its potential intranasal DA delivery by an AlgOX-based hydrogel formulation as an innovative PD treatment approach. It should be taken account that (i) intranasal administration may constitute a valuable non-invasive strategy to bypass the BBB hurdle; (ii) hydrogels have attracted considerable attention for intranasal administration; and (iii) the SH-SY5Y cell line has been used as a suitable experimental model for studying PD.

The conjugate AlgOX-DA herein proposed for nose-to-brain delivery was obtained after initial NaAlg oxidation according to [34]. Using Raman spectra, the oxidation of starting Alg occurred to produce AlgOX (Figure 1). Regarding the identification of AlgOX, as mentioned in Section 2.3, we assigned the structure of this polymer by comparison with the FT-IR spectrum reported in literature for an authentic sample (Figure 6). In particular, in this spectrum, the stretching absorption band of the aldehyde group was not detected, however, this is in agreement with literature reports [24]. It has been shown that aldehyde adsorption is not observed in dry samples of AlgOX-DA, while it can be detected in moist samples equilibrated at r.t. In this case, an equilibrium between aldehyde and hemiacetal groups occurs [24]. Concerning the structural assignment of the AlgOX-DA imine conjugate, once again, we did not observe the absorption band of the imine group expected in the range $1640-1690 \mathrm{~cm}^{-1}$ in the corresponding FT-IR spectrum. Although for AlgOX-DA the large band at $1636 \mathrm{~cm}^{-1}$ in the FT-IR spectrum can also be interpreted as the absorption of carboxylate groups of AlgOX overlapping with the Schiff base, the bands in the FT-IR spectrum help in the interpretation of the chemical structure of the conjugate AlgOX-DA. In this last spectrum, it is possible to detect a weak absorption band at $2915 \mathrm{~cm}^{-1}$ and $2917 \mathrm{~cm}^{-1}$ for AlgOX and AlgOX-DA, respectively, which may be attributed to the $\mathrm{H}-\mathrm{C}$ absorption of the aldehyde group, which is present in the chemical formulas of both the substances. On the other hand, the UV-vis and the ${ }^{1} \mathrm{H}-\mathrm{NMR}$ spectra clearly support the 
structural assignment made, particularly the latter spectrum, where the resonance of the imine group was detected at $7.5 \mathrm{ppm}$. The presence of peaks that can be ascribed to the DA moiety are in the range of 6.6-6.8 ppm (aromatic portion of DA) and 2.74-3.10 ppm (aliphatic portion of DA). It should be pointed out that the chemical shift of the imine group we found resulted in a good agreement with that reported in the literature for other $-\mathrm{CH}=\mathrm{N}$ containing conjugates. Moreover, elemental analysis combined to EDX also confirmed the composition of the conjugate AlgOX-DA and SEM microphotographs evidenced a similar external structure between pristine NaAlg and AlgOX-DA. In good agreement with such evidence, XPS data acquisition and spectra processing (Figure 9 and Table 1), following protocols that have been previously described [43], also led to the conclusion that the synthesis of the imine based conjugate AlgOX-DA was successfully carried out.

Furthermore, the DS of the final AlgOX-DA was achieved following either NMR calculations on the corresponding monodimensional spectrum or performing acid-induced hydrolysis followed by HPLC quantification of the delivered neurotransmitter. Of note, the DS of AlgOX-DA determined after chemical hydrolysis allowed quantitative evaluations and, therefore, DS was employed for in vitro release studies and AlgOX-DA exposure to the cell line. For instance, for AlgOX-DA, the DS for DA we found was almost double that previously studied for a Alg hydrogel containing DA, namely $15.86 \pm 2.04 \mu \mathrm{g}$ DA $/ \mathrm{mg}$ DA-hydrogel already studied for drug delivery applications [38].

In order to explore the potential of AlgOX and AlgOX-DA for nasal adaptability, in vitro investigations focusing on mucoadhesion and release were carried out (Figure 10). Turbidimetric measurements were carried out in the presence of a positive control of hydroxyethyl cellulose (HEC) because Carbopol 940 precipitated in SNF medium, as previously shown [44]. Of note, during mucoadhesion studies referring to AlgOX and AlgOXDA, no color change at any time of incubation was evidenced for both biomaterials, different from our previous investigations on another polymeric prodrug of DA; namely, a DA-ester-derivative of $\mathrm{N}, \mathrm{O}$-carboxymethyl chitosan, where at the last time point showed a black color in the SNF medium. Although the polymeric backbone is different (i.e., $\mathrm{N}, \mathrm{O}$-carboxymethyl chitosan vs. AlgOX), our hypothesis is that for both conjugates, during exposure to SNF, an autoxidation process of the neurotransmitter took place [28] once DA was released after hydrolytic cleavage in SNF. It might be faster for ester derivatives rather than for imine-based AlgOX-DA.

Interestingly, in vitro mucoadhesive studies in SNF showed that the conjugate AlgOXDA was more mucoadhesive than unmodified AlgOX, determining a reduction in percentage of transmittance of up to $78 \%$ within $24 \mathrm{~h}$ of mucin/AlgOX-DA contact (Figure 10). Such behavior can be explained in terms of the catechol moiety of the neurotransmitter that, in the AlgOX-DA backbone, improves the mucoadhesive effects. Chitosan-catecholcontaining conjugates have already been described for the enhancement of catechol moiety in terms of mucoadhesion $[45,46]$ and it seems that the same role could be exerted here, irrespective of the fact that the backbone is Alg-based. Such findings also seem in good agreement with Nur et al.'s investigations [47], evidencing that the prediction of mucoadhesive effects based on rheology measurements indicated that, among mucin-polymer mixtures, chitosan and polyvinyl pyrrolidone (PVP) exhibited a lower strength of mucoadhesion compared to Alg and its derivatives. On the other hand, for AlgOX-DA, such a high mucoadhesive effect is combined with a sustained release capability, as shown in Figure 11 a, where the hydrolysis of the Schiff base leads to a delivery of $13 \%$ of the neurotransmitter in $24 \mathrm{~h}$ in SNF, in agreement with the sensitivity of the Schiff base to undergo the hydrolysis process under acidic conditions. Nevertheless, free DA may slowly be further converted into degradation products and, therefore, we studied these consecutive reactions from a kinetic point of view according to Juriga et al.'s approach [48] for polymer-DA conjugates. In particular, for poorly soluble conjugates, such as the AlgOX-DA imine conjugate, DA release can be expressed by the diffusion of DA from the solid surface of the conjugate combined with the degradation reactions of DA in solution [48]. In the case of 
the AlgOX-DA imine conjugate, a $\mathrm{K}_{\mathrm{H}}$ value of $7.476 \pm 1.828\left(\%\right.$ of DA released $\times \mathrm{h}^{-1 / 2}$ ) for the first $3 \mathrm{~h}$ of release was calculated, indicative of a sustained neurotransmitter release.

Another critical feature for a DA-loaded formulation intended for nose-to-brain delivery for Parkinsonian patients is that the neurotransmitter is released in the dosage form in sustained manner so that a reduced stimulation of dopaminergic neurons occurs [28]. As shown in Section 3.8, in SNF, the neurotransmitter is released from the AlgOX-DA imine conjugate in a sustained fashion (as proved by the observed $\mathrm{K}_{\mathrm{H}}$ value) within the first $3 \mathrm{~h}$, even though it is about $13 \%$ of the total amount. This low amount of neurotransmitter released in a sustained manner, may even be advantageous for the decreased neuronal toxicity [28]. Moreover, it should be also considered that (i) DA, as neurotransmitter, is a potent substance able to produce its biological effects, even if present in small amounts; (ii) the conditions we used for these in vitro release studies cannot be considered fully biomimetic and, hence, definitive information on DA release from AlgOX-DA imine conjugate can only be gained by performing in vivo studies. On the other hand, when PBS was used as a receiving medium for in vitro release tests (Figure 11c), its physiological $\mathrm{pH}$ value is of aid to understand that the clear decrease in neurotransmitter release observed at longer times should likely be ascribed to the most likely autoxidation reaction of the neurotransmitter in neutral/alkaline conditions. Preliminary tests of AlgOX-DA stability in fetal bovine serum/PBS $(1: 1, v / v)$ were started by us in order to predict the behavior of the conjugate once absorbed by blood vessels (unpublished data). Three percent of DA was found to be delivered within $3 \mathrm{~h}$ and, once data are replicated, such findings could mean that an almost intact polymeric prodrug AlgOX-DA could by distributed from capillary vessels to the Substantia Nigra in the brain, providing a site-specific delivery of the neurotransmitter.

Overall, delivery systems allowing sustained release of a neurotransmitter, as seen for AlgOX-DA, may be useful to avoid multiple administrations of L-Dopa, which is currently the gold standard for PD treatment. In addition, if a sustained release of DA can be achieved in vivo by replacing L-Dopa administrations [49], a reduction in the toxic effects of L-Dopa can be expected, due to the fact that they typically emerge at later stages of PD. Overall, in view of nose-to-brain delivery, mean particle size of AlgOX-DA (Table 2) seems promising for bypassing the BBB because, as with colloidal carriers aiming at such non-invasive absorption approach, it has already been discussed that $200 \mathrm{~nm}$ is regarded as a suitable value for mean diameter [50,51].

From a biological viewpoint, it is well known that DA induces a loss of cell viability associated with mitochondrial dysfunction in SH-SY5Y cells [52], thus the strategy of bioconjugation was selected by us in an attempt to overcome such limitations. Therefore, in view of cell/conjugate interaction studies, fluorescent dye FITC was condensed on AlgOX and cell viability studies with SH-SY5Y (Figure 13b) evidenced that FITC-AlgOX can be considered a safe probe material if incubated for $24 \mathrm{~h}$ up to a concentration of $450 \mathrm{~g} / \mathrm{mL}$, whereas unlabeled AlgOX showed cytotoxicity at $400 \mathrm{~g} / \mathrm{mL}$ (Figure 13a).

Concerning the microscopic evaluation of FITC-AlgOX, the SNOM apparatus employed worked with a resolution that depended on the aperture dimension of the optical fiber rather than the wavelength of the light, as is the case in conventional optical fluorescence spectroscopy. Therefore, only a small volume of the sample was optically illuminated, in order to minimize fluorophore bleaching and, consequently, the biological damage normally occurring in optical fluorescence microscopy was reduced. Herein, the use of a localized optical microscope, such as SNOM, allowed a deep investigation in the features of fluorescently labeled AlgOX samples, focusing on targeted areas of the sample. The SNOM characterization of the FITC-AlgOX fluorescent effect (Figure 12) evidenced a homogeneous distribution within the conjugate, either at $1 \mathrm{mg} / \mathrm{mL}$ or $0.01 \mathrm{mg} / \mathrm{mL}$ concentrations, which were safe concentrations of FITC-AlgOX upon incubation with SH-SY5Y cell lines.

Generally, biological samples are characterized by their intrinsic variability, such as differences in shape or functionality in the same cell culture population. The SNOM microscope is well known to provide high-resolution optical images, including nanometer-scale topographic information [53-55], and is of aid in the examination of biological interac- 
tions, allowing a fast and direct identification and analysis of specific features. Finally, SNOM images and characterization of FITC-AlgOX alone obtained herein will be available for further studies, aiming at monitoring topographical and optical characteristics upon cell incubation.

\section{Conclusions}

In this work, investigations focusing on the conjugation of DA via an imine bond to AlgOX were performed. Multiple spectroscopic techniques, namely, ${ }^{1} \mathrm{H}$ NMR, UV-vis, FT-IR and X-ray photoelectron spectroscopic methods, confirmed the presence of the Schiff base for the AlgOX-Da conjugate and the slow release of DA was revealed after incubation in SNF and in PBS. The conjugate showed mucoadhesive properties in vitro that were better than those of pristine polymer Alg, as well as a sustained release of DA in SNF at about $13 \%$; an amount that may be advantageous for a decrease in neuronal toxicity and sufficient to perform its biological effects given the high potency of the neurotransmitter. Furthermore, the cytocompatibility of the imine conjugate was assessed using the SRB assay against human neuroblastoma SH-SY5Y cells in a wide range of concentrations. Our work demonstrates that the AlgOX-DA imine conjugate is a promising delivery system for non-invasive nasal administration, as an innovative approach for PD treatment, using a hydrogel dosage form or polymeric nanoparticles [43].

Author Contributions: Data curation, N.D., N.C., P.L., A.M. and S.M.; formal analysis, E.S.; investigation, A.Q., A.C. (Antonio Cricenti), C.M. and A.C. (Antonino Cataldo); methodology F.C. and G.A.; software, F.P.; validation M.L.; supervision, A.T. and S.B. All the authors contributed to the discussion and reviewed the manuscript. All authors have read and agreed to the published version of the manuscript.

Funding: This research received no external funding.

Institutional Review Board Statement: Experiments were carried out in compliance with the Italian law on animal care no.116/1992 and in accordance with the European Community CouncilDirective (86/609/EEC).

Informed Consent Statement: Not applicable.

Data Availability Statement: The data presented in this study are available on request from the corresponding authors. The data are not publicly available due to ethic reasons.

Acknowledgments: A.T. would acknowledge Mauro Coluccia and Alessandra Pannunzio (University of Bari, Italy) for their valuable technical assistance. A.C. (Antonino Cataldo) and S.B. would also acknowledge Emanuele Mauri (Rome-Campus Biomedico, Italy), P. Tagliatesta and Alessandro Leoni (Rome-Tor Vergata University, Italy) for their support in the experimental setup. This work is dedicated to Saverio Cellamare on the occasion of his retirement.

Conflicts of Interest: The authors declare no conflict of interest.

\section{References}

1. Di Stefano, A.; Sozio, P.; Iannitelli, A.; Cerasa, L.S. New drug delivery strategies for improved Parkinson's disease therapy. Expert Opin. Drug Deliv. 2009, 6, 389-404. [CrossRef]

2. Levin, J.; Schmidt, F.; Boehm, C.; Prix, C.; Bötzel, K.; Ryazanov, S.; Leonov, A.; Griesinger, C.; Giese, A. The oligomer modulator anle138b inhibits disease progression in a Parkinson mouse model even with treatment started after disease onset. Acta Neuropathol. 2014, 127, 779-780. [CrossRef] [PubMed]

3. Saraiva, C.; Praça, C.; Ferreira, R.; Santos, T.; Ferreira, L.; Bernardino, L. Nanoparticle-mediated brain drug delivery: Overcoming blood-brain barrier to treat neurodegenerative diseases. J. Control. Release 2016, 235, 34-47. [CrossRef] [PubMed]

4. Agrawal, M.; Saraf, S.; Saraf, S.; Antimisiaris, S.G.; Chougule, M.B.; Shoyele, S.A.; Alexander, A. Nose-to-brain drug delivery: An update on clinical challenges and progress towards approval of anti-Alzheimer drugs. J. Control. Release 2018, 281, 139-177. [CrossRef] [PubMed]

5. Bourganis, V.; Kammona, O.; Alexopoulos, A.; Kiparissides, C. Recent advances in carrier mediated nose-to-brain delivery of pharmaceutics. Eur. J. Pharm. Biopharm. 2018, 128, 337-362. [CrossRef]

6. Samaridou, E.; Alonso, M.J. Nose-to-brain peptide delivery-The potential of nanotechnology. Bioorganic Med. Chem. 2018, 26, 2888-2905. [CrossRef] 
7. Gänger, S.; Schindowski, K. Tailoring Formulations for Intranasal Nose-to-Brain Delivery: A Review on Architecture, PhysicoChemical Characteristics and Mucociliary Clearance of the Nasal Olfactory Mucosa. Pharmaceutics 2018, 10, 116. [CrossRef]

8. Mandracchia, D.; Trapani, A.; Perteghella, S.; Sorrenti, M.; Catenacci, L.; Torre, M.L.; Trapani, G.; Tripodo, G. pH-Sensitive Inulin-Based Nanomicelles for Intestinal Site-Specific and Controlled Release of Celecoxib. Carbohydr. Polym. 2018, 181, 570-578. [CrossRef] [PubMed]

9. Mandracchia, D.; Trapani, A.; Tripodo, G.; Perrone, M.G.; Giammona, G.; Trapani, G.; Colabufo, N.A. In vitro evaluation of glycol chitosan based formulations as oral delivery systems for efflux pump inhibition. Carbohydr. Polym. 2017, 166, 73-82. [CrossRef]

10. Tripodo, G.; Trapani, A.; Rosato, A.; Di Franco, C.; Tamma, R.; Ribatti, D.; Mandracchia, D. Hydrogels for biomedical applications from glycol chitosan and PEG diglycidyl ether exhibit pro-angiogenic and antibacterial activity. Carbohydr. Polym. 2018, 198, 124-130. [CrossRef]

11. Sonvico, F.; Clementino, A.; Buttini, F.; Colombo, G.; Pescina, S.; Guterres, S.S.; Pohlmann, A.R.; Nicoli, S. Surface-Modified Nanocarriers for Nose-to-Brain Delivery: From Bioadhesion to Targeting. Pharmaceutics 2018, 10, 34. [CrossRef]

12. Clementino, A.; Batger, M.; Garrastazu, G.; Pozzoli, M.; Del Favero, E.; Rondelli, V.; Gutfilen, B.; Barboza, T.; Sukkar, M.B.; Souza, S.A.; et al. The Nasal Delivery of Nanoencapsulated Statins-an Approach for Brain Delivery. Int. J. Nanomed. 2016, 11, 6575-6590. [CrossRef] [PubMed]

13. Sorrentino, A.; Cataldo, A.; Curatolo, R.; Tagliatesta, P.; Mosca, L.; Bellucci, S. Novel optimized biopolymer-based nanoparticles for nose-to-brain delivery in the treatment of depressive diseases. RSC Adv. 2020, 10, 28941-28949. [CrossRef]

14. Ghazy, E.; Rahdar, A.; Barani, M.; Kyzas, G.Z. Nanomaterials for Parkinson disease: Recent progress. J. Mol. Struct. 2021, 1231, 129698. [CrossRef]

15. De Giglio, E.; Trapani, A.; Cafagna, D.; Sabbatini, L.; Cometa, S. Dopamine-loaded chitosan nanoparticles: Formulation and analytical characterization. Anal. Bioanal. Chem. 2011, 400, 1997-2002. [CrossRef]

16. Pahuja, R.; Seth, K.; Shukla, A.; Shukla, R.K.; Bhatnagar, P.; Chauhan, L.K.S.; Saxena, P.N.; Arun, J.; Chaudhari, B.P.; Patel, D.K.; et al. Trans-Blood Brain Barrier Delivery of Dopamine-Loaded Nanoparticles Reverses Functional Deficits in Parkinsonian Rats. ACS Nano 2015, 9, 4850-4871. [CrossRef] [PubMed]

17. Pillay, S.; Pillay, V.; Choonara, Y.E.; Naidoo, D.; Khan, R.A.; du Toit, L.; Ndesendo, V.M.; Modi, G.; Danckwerts, M.P.; Iyuke, S.E. Design, biometric simulation and optimization of a nano-enabled scaffold device for enhanced delivery of dopamine to the brain. Int. J. Pharm. 2009, 382, 277-290. [CrossRef] [PubMed]

18. Trapani, A.; De Giglio, E.; Cafagna, D.; Denora, N.; Agrimi, G.; Cassano, T.; Gaetani, S.; Cuomo, V.; Trapani, G. Characterization and evaluation of chitosan nanoparticles for dopamine brain delivery. Int. J. Pharm. 2011, 419, 296-307. [CrossRef]

19. Ekladious, I.; Colson, Y.L.; Grinstaff, M.W. Polymer-drug conjugate therapeutics: Advances, insights and prospects. Nat. Rev. Drug Discov. 2019, 18, 273-294. [CrossRef]

20. Mandracchia, D.; Rosato, A.; Trapani, A.; Chlapanidas, T.; Montagner, I.M.; Perteghella, S.; Di Franco, C.; Torre, M.L.; Trapani, G.; Tripodo, G. Design, synthesis and evaluation of biotin decorated inulin-based polymeric micelles as long-circulating nanocarriers for targeted drug delivery. Nanomed. Nanotechnol. Biol. Med. 2017, 13, 1245-1254. [CrossRef]

21. Trapani, A.; Palazzo, C.; Contino, M.; Perrone, M.G.; Cioffi, N.; Ditaranto, N.; Colabufo, N.A.; Conese, M.; Trapani, G.; Puglisi, G. Mucoadhesive Properties and Interaction with P-Glycoprotein (P-gp) of Thiolated-Chitosans and -Glycol Chitosans and Corresponding Parent Polymers: A Comparative Study. Biomacromolecules 2014, 15, 882-893. [CrossRef]

22. Hawthorne, G.H.; Bernuci, M.P.; Bortolanza, M.; Tumas, V.; Issy, A.C.; Del Bel, E. Nanomedicine to Overcome Current Parkinson's Treatment Liabilities: A Systematic Review. Neurotox. Res. 2016, 30, 715-729. [CrossRef] [PubMed]

23. Li, X.; Tsibouklis, J.; Weng, T.; Zhang, B.; Yin, G.; Feng, G.; Cui, Y.; Savina, I.; Mikhalovska, L.I.; Sandeman, S.R.; et al. Nano carriers for drug transport across the blood-brain barrier. J. Drug Target. 2017, 25, 17-28. [CrossRef] [PubMed]

24. Reakasame, S.; Boccaccini, A.R. Oxidized Alginate-Based Hydrogels for Tissue Engineering Applications: A Review. Biomacromolecules 2018, 19, 3-21. [CrossRef] [PubMed]

25. Pawar, S.N.; Edgar, K.J. Alginate derivatization: A review of chemistry, properties and applications. Biomaterials 2012, 33, 3279-3305. [CrossRef]

26. Gomez, C.; Rinaudo, M.; Villar, M. Oxidation of sodium alginate and characterization of the oxidized derivatives. Carbohydr. Polym. 2007, 67, 296-304. [CrossRef]

27. Gao, C.; Tang, F.; Gong, G.; Zhang, J.; Hoi, M.P.; Lee, S.M.; Wang, R. PH-Responsive Prodrug Nanoparticles Based on a Sodium Alginate Derivative for Selective Co-Release of Doxorubicin and Curcumin into Tumor Cells. Nanoscale 2017, 9 , 12533-12542. [CrossRef]

28. Cassano, R.; Trapani, A.; Di Gioia, M.L.; Mandracchia, D.; Pellitteri, R.; Tripodo, G.; Trombino, S.; Di Gioia, S.; Conese, M. Synthesis and characterization of novel chitosan-dopamine or chitosan-tyrosine conjugates for potential nose-to-brain delivery. Int. J. Pharm. 2020, 589, 119829. [CrossRef]

29. Pagar, S.A.; Shinkar, D.M.; Saudagar, R.B. Development and Evaluation of in Situ Nasal Mucoadhesive Gel of Metoprolol Succinate by Using 32 Full Factorial Design. Int. J. Pharm. Pharm. Sci. 2014, 16, 218-223.

30. Tan, M.-E.; He, C.-H.; Jiang, W.; Zeng, C.; Yu, N.; Huang, W.; Gao, Z.-G.; Xing, J.-G. Development of solid lipid nanoparticles containing total flavonoid extract from Dracocephalum moldavica L. and their therapeutic effect against myocardial ischemiareperfusion injury in rats. Int. J. Nanomed. 2017, 12, 3253-3265. [CrossRef] 
31. Di Gioia, S.; Trapani, A.; Mandracchia, D.; De Giglio, E.; Cometa, S.; Mangini, V.; Arnesano, F.; Belgiovine, G.; Castellani, S.; Pace, L.; et al. Intranasal delivery of dopamine to the striatum using glycol chitosan/sulfobutylether- $\beta$-cyclodextrin based nanoparticles. Eur. J. Pharm. Biopharm. 2015, 94, 180-193. [CrossRef] [PubMed]

32. Longo, G.; Girasole, M.; Pompeo, G.; Generosi, R.; Luce, M.; Cricenti, A. An inverted/scanning near-field optical microscope for applications in materials science and biology. Phys. Status Solidi B 2010, 247, 2051-2055. [CrossRef]

33. Kristiansen, K.A.; Potthast, A.; Christensen, B.E. Periodate oxidation of polysaccharides for modification of chemical and physical properties. Carbohydr. Res. 2010, 345, 1264-1271. [CrossRef]

34. Balakrishnan, B.; Lesieur, S.; Labarre, D.; Jayakrishnan, A. Periodate oxidation of sodium alginate in water and in ethanol-water mixture: A comparative study. Carbohydr. Res. 2005, 340, 1425-1429. [CrossRef] [PubMed]

35. Resmi, R.; Parvathy, J.; John, A.; Joseph, R. Injectable self-crosslinking hydrogels for meniscal repair: A study with oxidized alginate and gelatin. Carbohydr. Polym. 2020, 234, 115902. [CrossRef]

36. Campos-Vallette, M.M.; Chandía, N.P.; Clavijo, E.; Leal, D.; Matsuhiro, B.; Osorio-Román, I.O.; Torres, S. Characterization of sodium alginate and its block fractions by surface-enhanced Raman spectroscopy. J. Raman Spectrosc. 2009, 41, 758-763. [CrossRef]

37. Yue, Q.; Wang, M.; Sun, Z.; Wang, C.; Wang, C.; Deng, Y.; Zhao, D. A versatile ethanol-mediated polymerization of dopamine for efficient surface modification and the construction of functional core-shell nanostructures. J. Mater. Chem. B 2013, 1, 6085-6093. [CrossRef]

38. Gao, B.; Chen, L.; Zhao, Y.; Yan, X.; Wang, X.; Zhou, C.; Shi, Y.; Xue, W. Methods to prepare dopamine/polydopamine modified alginate hydrogels and their special improved properties for drug delivery. Eur. Polym. J. 2019, 110, 192-201. [CrossRef]

39. Sarmento, B.; Ferreira, D.; Veiga, F.; Ribeiro, A. Characterization of insulin-loaded alginate nanoparticles produced by ionotropic pre-gelation through DSC and FTIR studies. Carbohydr. Polym. 2006, 66, 1-7. [CrossRef]

40. Ivarsson, D.; Wahlgren, M. Comparison of in vitro methods of measuring mucoadhesion: Ellipsometry, tensile strength and rheological measurements. Colloids Surfaces B Biointerfaces 2012, 92, 353-359. [CrossRef]

41. Umek, N.; Geršak, B.; Vintar, N.; Šoštarič, M.; Mavri, J. Dopamine Autoxidation Is Controlled by Acidic pH. Front. Mol. Neurosci. 2018, 11, 467. [CrossRef]

42. Cricenti, A.; Generosi, R.; Luce, M.; Talley, D.; Sanghera, J.S.; Perfetti, P.; Margaritondo, G.; Aggarwal, I.D.; Tolk, N.H. Very high resolution near-field chemical imaging using an infrared free electron laser. Phys. Chem. Chem. Phys. 2002, 4, 2738-2741. [CrossRef]

43. Ancona, A.; Sportelli, M.; Trapani, A.; Picca, R.; Palazzo, C.; Bonerba, E.; Mezzapesa, F.; Tantillo, G.M.; Trapani, G.; Cioffi, N. Synthesis and characterization of hybrid copper-chitosan nano-antimicrobials by femtosecond laser-ablation in liquids. Mater. Lett. 2014, 136, 397-400. [CrossRef]

44. Trapani, A.; Giglio, E.D.; Cometa, S.; Bonifacio, M.A.; Dazzi, L.; Di Gioia, S.; Hossain, M.N.; Pellitteri, R.; Antimisiaris, S.G.; Conese, M. Dopamine-Loaded Lipid Based Nanocarriers for Nose-to-Brain Delivery of the Neurotransmitter: A Comparative Study. Eur. J. Pharm. Biopharm. 2021, under review.

45. Kim, K.; Kim, K.; Ryu, J.H.; Lee, H. Chitosan-Catechol: A Polymer with Long-Lasting Mucoadhesive Properties. Biomaterials 2015, 52, 161-170. [CrossRef]

46. Ryu, J.H.; Hong, S.; Lee, H. Bio-inspired adhesive catechol-conjugated chitosan for biomedical applications: A mini review. Acta Biomater. 2015, 27, 101-115. [CrossRef] [PubMed]

47. Nur, M.; Ramchandran, L.; Vasiljevic, T. Tragacanth as an oral peptide and protein delivery carrier: Characterization and mucoadhesion. Carbohydr. Polym. 2016, 143, 223-230. [CrossRef] [PubMed]

48. Juriga, D.; Laszlo, I.; Ludanyi, K.; Klebovich, I.; Chae, C.H.; Zrinyi, M. Kinetics of dopamine release from poly(aspartamide)-based prodrugs. Acta Biomater. 2018, 76, 225-238. [CrossRef]

49. Rodríguez-Nogales, C.; Garbayo, E.; Carmona-Abellán, M.; Luquin, M.; Blanco-Prieto, M. Brain aging and Parkinson's disease: New therapeutic approaches using drug delivery systems. Maturitas 2016, 84, 25-31. [CrossRef]

50. Mistry, A.; Glud, S.Z.; Kjems, J.; Randel, J.; Howard, K.A.; Stolnik, S.S.; Illum, L. Effect of physicochemical properties on intranasal nanoparticle transit into murine olfactory epithelium. J. Drug Target. 2009, 17, 543-552. [CrossRef]

51. Trapani, A.; Guerra, L.; Corbo, F.; Castellani, S.; Sanna, E.; Capobianco, L.; Monteduro, A.; Manno, D.; Mandracchia, D.; Di Gioia, S.; et al. Cyto/Biocompatibility of Dopamine Combined with the Antioxidant Grape Seed-Derived Polyphenol Compounds in Solid Lipid Nanoparticles. Molecules 2021, 26, 916. [CrossRef] [PubMed]

52. Ganguly, U.; Ganguly, A.; Sen, O.; Ganguly, G.; Cappai, R.; Sahoo, A.; Chakrabarti, S. Dopamine Cytotoxicity on SH-SY5Y Cells: Involvement of $\alpha$-Synuclein and Relevance in the Neurodegeneration of Sporadic Parkinson's Disease. Neurotox. Res. 2019, 35 , 898-907. [CrossRef]

53. Cricenti, A.; Generosi, R.; Luce, M.; Perfetti, P.; Margaritondo, G.; Talley, D.; Sanghera, J.S.; Aggarwal, I.D.; Tolk, N.H.; Congiu-Castellano, A.; et al. Chemically Resolved Imaging of Biological Cells and Thin Films by Infrared Scanning Near-Field Optical Microscopy. Biophys. J. 2003, 85, 2705-2710. [CrossRef]

54. Rieti, S.; Manni, V.; Lisi, A.; Giuliani, L.; Sacco, D.; D’Emilia, E.; Cricenti, A.; Generosi, R.; Luce, M.; Grimaldi, S. SNOM and AFM microscopy techniques to study the effect of non-ionizing radiation on the morphological and biochemical properties of human keratinocytes cell line (HaCaT). J. Microsc. 2003, 213, 20-28. [CrossRef] [PubMed]

55. Sanghera, J.S.; Aggarwal, I.D.; Cricenti, A.; Generosi, R.; Luce, M.; Perfetti, P.; Margaritondo, G.; Tolk, N.H.; Piston, D. Infrared Scanning Near-Field Optical Microscopy Below the Diffraction Limit. IEEE J. Sel. Top. Quantum Electron. 2008, 14, 1343-1352. [CrossRef] 